\title{
Lifetime of kaonium
}

\author{
S. Krewald, R. H. Lemmer, * and F. P. Sassen \\ Institut für Kernphysik, Forschungszentrum Jülich GmbH, D-52425 Jülich, Germany
}

(Received 23 July 2003; published 29 January 2004)

\begin{abstract}
The kaon-antikaon system is studied in both the atomic and the strongly interacting sector. We discuss the influence of the structures of the $f_{0}(980)$ and the $a_{0}(980)$ mesons on the lifetime of kaonium. The strong interactions are generated by vector meson exchange within the framework of the standard $S U(3)_{V}$ $\times S U(3)_{A}$ invariant effective Lagrangian. In the atomic sector, the energy levels and decay widths of kaonium are determined by an eigenvalue equation of the Kudryavtsev-Popov type, with the strong interaction effects entering through the complex scattering length for $K \bar{K}$ scattering and annihilation. The presence of two scalar mesons $f_{0}(980)$ and $a_{0}(980)$ leads to a ground state energy for the kaonium atom that is shifted above the point Coulomb value by a few hundred $\mathrm{eV}$. The effect on the lifetime for the kaonium decay into two pions is much more dramatic. This lifetime is reduced by two orders of magnitude from $1.2 \times 10^{-16} \mathrm{sec}$ for annihilation in a pure Coulomb field down to $3.2 \times 10^{-18} \mathrm{sec}$ when the strong interactions are included. The analysis of the two photon decay width of the $f_{0}(980)$ suggests a generalization of the molecular picture which reduces the lifetime of kaonium still further to $1.1 \times 10^{-18} \mathrm{sec}$.
\end{abstract}

DOI: 10.1103/PhysRevD.69.016003 PACS number(s): 11.10.St, 14.40.Aq, 14.40.Cs, 36.10.-k

\section{INTRODUCTION}

There has been substantial experimental progress in the field of meson spectroscopy during the last decade [1]. In the energy region up to $2 \mathrm{GeV}$, more scalar-isoscalar mesons have been established than can be accounted for by a quarkantiquark structure [2]. The structure of the scalar meson with the lowest mass, $f_{0}(980)$, has been controversial for many years. The $f_{0}(980)$ might be a $q \bar{q}$ state $[3,4]$, a $q^{2} \bar{q}^{2}$ state [5], or a $K \bar{K}$ molecule [6].

The radiative decay of the $\phi$ meson provides a particularly strong argument for a $q^{2} \bar{q}^{2}$ interpretation of both the $f_{0}(980)$ and the $a_{0}(980)$ mesons, as was first pointed out by Achasov and Ivanchenko [5]. Both the recent Novosibirsk data [7] and the KLOE data [8] can be reproduced by a calculation which generates those mesons dynamically, however [9]. Since in Ref. [9] Oller had to introduce a $\phi$ $\rightarrow \gamma K^{0} \bar{K}^{0}$ contact interaction, the issue remains controversial [10].

The production of two neutral pions in ultrarelativistic pion proton reactions shows a strong dependence of the $s$-wave amplitude on the momentum transferred between the proton and the neutron for invariant two-pion masses in the vicinity of $1 \mathrm{GeV}[11,12]$. This fact has been interpreted as evidence for a hard component of the $f_{0}(980)$, see e.g. Ref. [13]. A recent calculation based on a model which allows for a dynamical generation of the $f_{0}(980)$ provides a good description of the data [14]. On the other hand, the model employed in Ref. [14] also includes a bare scalar resonance. Given this situation, we feel that a simplified calculation might be helpful.

In this paper, we develop an analytical model which gen-

\footnotetext{
*Permanent address: Nuclear and Particle Theory Group, University of the Witwatersrand, Johannesburg, Private Bag 3, WITS 2050, South Africa.
}

erates the $f_{0}(980)$ meson as a bound $K \bar{K}$ structure. This model makes specific predictions for the structure of the exotic atom kaonium. In a second step, we work out the predictions for kaonium based on the meson-exchange model of Ref. [14].

The molecular interpretation is consistent with the small binding energy $\sim 10-20 \mathrm{MeV}$ of the $K \bar{K}$ system relative to its reduced mass $M_{K} \approx 496 \mathrm{MeV}$. This suggests a nonrelativistic effective field theory approach [15] that has also been used recently to study both pionium $[16,17]$ as well as $O\left(\alpha^{6}\right)$ QED recoil and radiative corrections to the positronium spectrum [18]. With this in mind we use the standard $S U(3)_{V} \times S U(3)_{A}$ Lagrangian [19] to describe the dynamics of the $K \bar{K}$ interaction [20,21] and decay via the exchange of $\rho, \omega, \phi, K^{*} \ldots$ vector mesons. The coupling constants appearing in the Lagrangian are related via $S U(3)$ symmetry to the $\rho \pi \pi$ coupling constant $g_{\rho \pi \pi}$, which in turn can be obtained from the Kawarabayashi-Suzuki-RiazuddinFayyazuddin (KSRF) relation [22]. Thus once the decision on the form of Lagrangian has been taken, only physical meson masses and known coupling constants enter into the calculations.

We work in the nonrelativistic limit in which case two important simplifications occur: (i) only $t$-channel exchange diagrams survive, and (ii) the resulting one-meson exchange potentials become local. This in turn means that one can reduce the Bethe-Salpeter (BS) integral equation for the bound states of the interacting $K \bar{K}$ system to a local twobody Schrödinger wave equation that offers a significant simplification over working with integral equations [20,23].

After a brief recall of the derivation of the wave equation for nonrelativistic local potentials from the BS equation in Sec. II, the calculation of one-meson exchange potentials involving both direct $\rho, \omega, \phi$ transfer between $K$ and $\bar{K}$ as well as $K \bar{K} \rightarrow K \bar{K}$ scattering via two-pion intermediate states involving $K^{*}$ exchanges is carried out in Sec. III. We refer to 
these potentials collectively as one-boson exchange (OBE) potentials. The last contribution is essential to describe the $K \bar{K} \rightarrow 2 \pi$ decay channel. Then we make use of the fact that one can describe the low-energy properties of $K \bar{K}$ very adequately in the effective range approximation, to replace the OBE potentials by phase-equivalent potentials of the Bargmann type [24] that give rise to the same scattering length and effective range. These potentials offer the unique advantage of having known analytic solutions so that the associated Jost functions can be constructed explicitly.

A knowledge of the Jost functions in turn determines both the scattering and bound state properties of the $K \bar{K}$ system without further approximation [25]. In Sec. IV we carry out this program and compute both the mass and decay width of the kaonic molecule from the relevant Jost function that includes annihilation contributions. The resulting complex total energy for this system is $(981-25 i) \mathrm{MeV}$ that is in reasonable agreement with the recent experimental data from Fermilab [26] that give $[(975 \pm 3)-(22 \pm 2) i] \mathrm{MeV}$.

We also give computed elastic and reaction cross sections for $K \bar{K}$ scattering, the $\pi \pi$ inelasticities, and the cross section for the inverse process, $\pi^{+} \pi^{-} \rightarrow K \bar{K}$, for which data exist, using detailed balance. Those calculations disagree with the measured cross section, particularly near threshold [27-29].

On the other hand the similarity in the pole position in both the recent Fermilab measurements as well as the calculated position of this pole is striking. We also show that the molecular picture is totally inadequate for describing the 2 photon decay width of $f_{0}(980)$, which comes out to be an order of magnitude larger than experiment. Taken together with the overall underestimate of the $\pi^{+} \pi^{-} \rightarrow K \bar{K}$ data, this suggests that the $f_{0}(980)$ ground state cannot be a pure molecular state (see also [30]). This point is taken further at the end of Sec. IV.

We take up the discussion of kaonium in Sec. V. Since kaonium is a mixture of isoscalar and isovector states due to isospin-breaking introduced by the Coulomb potential, we appeal to the no-internal-mixing approximation that was introduced in connection with isobaric analog states in nuclei. In this approximation one joins linear combinations of the $I=0$ and $I=1$ isospin amplitudes in the external region where only the Coulomb field is important, onto (in this case) known solutions of good isospin of the wave equation in the internal region where the strong field is dominant. This allows one to construct the Jost function for kaonium without further approximation. Only momenta of $O\left(M_{K} \alpha\right)$, where $\alpha \approx 1 / 137$, are of interest for kaonium. This fact allows one to recast the condition that its bound states are given by the zeros of the Jost function in the lower half of the complex momentum plane as an eigenvalue equation of the Kudryavtsev-Popov type [31], in which the strong field effects only enter through the $K^{+} K^{-}$scattering length. The solutions of this equation show that the kaonium levels are shifted upwards from their pure Coulomb values and acquire lifetimes between $10^{-18}$ and $10^{-16} \mathrm{sec}$ in the presence of the $K \bar{K}$ molecular ground state.

We also show explicitly that the strong field introduces an additional node into the eigenfunctions of kaonium that lies at the $K^{+} K^{-}$scattering length. The kaonium levels are therefore to be viewed as excited states of the kaonic molecule in the combined Coulomb and strong fields of the system. This in turn has the effect of enhancing the $K^{+} K^{-} \rightarrow 2 \pi$ annihilation widths by two orders of magnitude over what they would have been under Coulomb binding of the charged kaon pair alone.

\section{FORMALISM}

In order to obtain a nonrelativistic equation for describing possible bound states of a $K \bar{K}$ meson pair resulting from vector meson exchange between them we briefly recall the derivation given by Landau and Lifshitz [32] that starts out with the four-point vertex $i \Gamma\left[p_{3}, p_{4} ; p_{1}, p_{2}\right]$ that enters the Bethe-Salpeter (BS) equation [33]. The $p_{1}, p_{2}$ and $p_{3}, p_{4}$ are incoming and outgoing meson four-momenta respectively. Then the homogeneous equation for this vertex that determines the bound state poles of the BS equation is

$$
\begin{aligned}
i \Gamma\left[p_{3}, p_{4} ; p_{1}, p_{2}\right]= & \int \frac{d^{4} q}{(2 \pi)^{4}} i \hat{\Gamma}\left[p_{3}, p_{4} ; q, p_{3}+p_{4}-q\right] \\
& \times(-i) D(q)(-i) D\left(p_{3}+p_{4}-q\right) \\
& \times i \Gamma\left[q, p_{3}+p_{4}-q ; p_{1} p_{2}\right] .
\end{aligned}
$$

All momentum labels formally flow from right to left, and the sum of each pair on either side of the semicolon equals the total four-momentum $P$ of the incoming pair which is conserved throughout the diagrammatic equation. The hatted vertex is the irreducible piece that generates $\Gamma$ by iteration. The $D$ 's are meson propagators. For a free meson of fourmomentum $q$ and mass $M$ one has

$$
-i D(q)=\frac{i}{q^{2}-M^{2}+i 0} \approx\left(\frac{1}{2 M}\right) \frac{i}{q_{0}-M-\mathbf{q}^{2} / 2 M+i 0}
$$

if in addition we move to the nonrelativistic limit by formally suppressing propagation "backwards" in time, i.e. by omitting the antiparticle pole in the upper half of the complex $q_{0}$ plane. We note [32] that the momenta $p_{1}, p_{2}$ are simply labels in Eq. (1) that are not determined by the equation at all. So one can simply drop them. The other simplification is to observe that the combination

$$
\chi\left(p_{3}, p_{4}\right)=D\left(p_{3}\right) \Gamma\left[p_{3}, p_{4}\right] D\left(p_{4}\right)
$$

appears under the integral sign. Thus one can equally well recast Eq. (1) as an integral equation for $\chi\left(p_{3}, p_{4}\right)$. This is most usefully written down in the center of mass $(\mathrm{CM})$ system for which $\left[p_{3}, p_{4}\right]= \pm p+P / 2$ with $p=\left[p_{0}, \mathbf{p}\right]$ and $P$ $=\left[P_{0}, 0\right]$, where $P_{0}$ is the total energy in the CM frame. Then the equation determining the bound states reads [32] 


$$
\begin{aligned}
i \chi(p, P)= & D\left(p+\frac{1}{2} P\right) D\left(-p+\frac{1}{2} P\right) \int \frac{d^{4} q}{(2 \pi)^{4}} \hat{\Gamma}\left[p+\frac{1}{2} P\right. \\
& \left.-p+\frac{1}{2} P ; q+\frac{1}{2} P,-q+\frac{1}{2} P\right] \chi(q, P)
\end{aligned}
$$

where $\chi\left(p_{3}, p_{4}\right)=\chi(p+P / 2,-p+P / 2) \equiv \chi(p, P)$.

To make further progress towards a nonrelativistic equation, the vertex $\hat{\Gamma}$ should not depend on the time components of the relative outgoing and incoming four-momenta $p$ $=\left[p_{0}, \mathbf{p}\right]$ and $q=\left[q_{0}, \mathbf{q}\right]$, i.e.

$$
\hat{\Gamma}\left[p+\frac{1}{2} P,-p+\frac{1}{2} P ; q+\frac{1}{2} P,-q+\frac{1}{2} P\right] \approx \hat{\Gamma}\left[\mathbf{p}, \mathbf{q}, P_{0}\right]
$$

Should this be the case one can then integrate out these time components from $\chi$ to obtain what is effectively the 3D Fourier transform of the wave function for relative motion,

$$
\psi(\mathbf{p})=\int_{-\infty}^{\infty} \frac{d p_{0}}{2 \pi} \chi(p, P)
$$

Carrying out the integrals over the time components of the relative four-momenta one finally arrives at the desired equation,

$$
\left(\frac{\mathbf{p}^{2}}{M}+2 M-P_{0}\right) \psi(\mathbf{p})-\int \frac{d^{3} \mathbf{q}}{(2 \pi)^{3}} \frac{\hat{\Gamma}\left[\mathbf{p}, \mathbf{q}, P_{0}\right]}{4 M^{2}} \psi(\mathbf{q})=0
$$

that makes use of the nonrelativistic approximation

$$
\begin{gathered}
\int_{-\infty}^{\infty} \frac{d p_{0}}{2 \pi i} D\left(p+\frac{1}{2} P_{0}\right) D\left(-p+\frac{1}{2} P_{0}\right) \\
\approx \frac{1}{4 M^{2}}\left(\frac{1}{\mathbf{p}^{2} / M+2 M-P_{0}}\right)
\end{gathered}
$$

as given by Eq. (2). One recognizes Eq. (7) as the Schrödinger wave equation in momentum space for relative motion in a potential

$$
V(\mathbf{p}, \mathbf{q})=-\frac{\hat{\Gamma}\left[\mathbf{p}, \mathbf{q}, P_{0}\right]}{4 M^{2}}
$$

and binding energy $\varepsilon=2 M-P_{0} \geqslant 0$. Should $\hat{\Gamma}\left[\mathbf{p}, \mathbf{q}, P_{0}\right]$ only depend on the difference $\mathbf{p}-\mathbf{q}$, the corresponding potential will be local in coordinate space. Since $\hat{\Gamma}$ is a relativistic proper vertex that in lowest order gives the $T$ matrix, the mass factor $1 / 4 M^{2}$ that converts $\hat{\Gamma}$ into a nonrelativistic potential is the same factor [16] that relates the relativistic $T$ matrix to its nonrelativistic counterpart.

\section{ONE-BOSON EXCHANGE POTENTIALS}

In the following we use Eq. (9) to investigate the possible binding of the $K \bar{K}$ system via one-boson exchange potentials by constructing $\hat{\Gamma}$ from the relevant pieces of the standard $S U(3)_{V} \times S U(3)_{A}$ invariant Lagrangian, the derivation and properties of which are described in detail in Ref. [19]. For $K \bar{K}$ scattering the relevant interaction Lagrangians for our purposes are

$$
\begin{aligned}
& \mathcal{L}_{K K \rho}=g_{K K \rho}\left[K^{\dagger} \vec{\tau} \partial_{\mu} K\right] \vec{\rho}^{\mu}+\text { c.c. } \\
& \mathcal{L}_{K K \omega}=g_{K K \omega}\left[K^{\dagger} \partial_{\mu} K\right] \omega^{\mu}+\text { c.c. } \\
& \mathcal{L}_{K K \phi}=g_{K K \phi}\left[K^{\dagger} \partial_{\mu} K\right] \phi^{\mu}+\text { c.c. }
\end{aligned}
$$

which, together with

$$
\mathcal{L}_{\pi K K^{*}}=g_{\pi K K^{*}}\left[\partial^{\mu} \vec{\pi} \cdot K^{\dagger} \vec{\tau} K_{\mu}^{*}-\vec{\pi} \cdot \partial^{\mu} K^{\dagger} \vec{\tau} K_{\mu}^{*}\right]+\text { c.c. }
$$

generate interaction potentials for $K \bar{K} \rightarrow K \bar{K}$ scattering via $\rho, \omega, \phi$ vector meson exchange, as well as for $K \bar{K} \rightarrow \pi \pi$ annihilation via $K^{*}(892)$ strange meson exchange. The $K$ 's are all isospin doublets and c.c. stands for the additional charge conjugation contribution with $K \rightarrow \bar{K}, K^{*} \rightarrow \bar{K}^{*}$.

The coupling constants in these expressions are all fixed in terms of the $\rho \pi \pi$ coupling constant $g_{\rho \pi \pi}$ by $S U(3)$ symmetry relations [19],

$$
\begin{gathered}
g_{K K \rho}=g_{\bar{K} \bar{K} \rho}=\frac{1}{2} g_{\rho \pi \pi} \\
g_{K K \omega}=g_{\bar{K} \bar{K} \omega}=-\frac{1}{2} g_{\rho \pi \pi} \\
g_{K K \phi}=g_{\bar{K} \bar{K} \phi}=\frac{1}{\sqrt{2}} g_{\rho \pi \pi} \\
g_{\pi K K^{*}}=g_{\pi \bar{K} \bar{K} *}=-\frac{1}{2} g_{\rho \pi \pi} .
\end{gathered}
$$

On the other hand the $\rho \pi \pi$ coupling is determined by the KSRF relation [22] as $g_{\rho \pi \pi} \approx M_{\rho} /\left(\sqrt{2} f_{\pi}\right) \approx 6$ in terms of the $\rho$ meson mass $M_{\rho}$ and the pion weak decay constant $f_{\pi}$ $\approx 93 \mathrm{MeV}$. In this sense, then, there are no free parameters in the calculation of the exchange potentials. They only contain physical meson masses and known coupling constants.

In the nonrelativistic limit, $p_{i}+p_{j} \approx\left[M_{i}+M_{j}, 0\right], p_{i}-p_{j}$ $\approx\left[0, \mathbf{p}_{i}-\mathbf{p}_{j}\right]$ only the $t$-channel scattering diagrams are relevant for determining $\hat{\Gamma}$. In this limit these amplitudes all have a common Yukawa-like form

$$
\begin{gathered}
\hat{\Gamma}\left[p+\frac{1}{2} P,-p+\frac{1}{2} P ; q+\frac{1}{2} P,-q+\frac{1}{2} P\right] \\
\approx g^{2} C_{I} n_{s} \frac{\left(M_{1}+M_{3}\right)\left(M_{2}+M_{4}\right)}{M^{2}+\mathbf{k}^{2}}
\end{gathered}
$$


in momentum space, where $\mathbf{k}=\mathbf{p}-\mathbf{q}$ is the $t$ channel 3-momentum transfer, and $M_{i}$ are the masses in the indicated entrance and exit channels; $M$ is the mass of the exchanged boson and $g$ the coupling constant. The isospin and boson identity factors $C_{I}$ and $n_{s}$ in Eq. (13) are given in [20]. They are $C_{0}=[3,1,1]$ and $C_{1}=[-1,1,1]$ and $n_{s}=1$ for $\rho, \omega, \phi$ exchange in the $K \bar{K} \rightarrow K \bar{K}$ isoscalar and isovector channels. The corresponding values for $K^{*}$ exchange in the $K \bar{K}$ $\rightarrow \pi \pi$ isoscalar and isovector channels are $C_{0}=-\sqrt{6}, C_{1}$ $=-2$, and $n_{s}=1 / \sqrt{2}$.

\section{A. $K \bar{K}$ exchange potentials}

Since $\hat{\Gamma}$ is only a function of the 3-momentum transfer, the resulting potentials in Eq. (9) are attractive local Yukawa potentials in coordinate space, and the determination of their possible bound states is numerically straightforward. While not necessary for convergence in the nonrelativistic case, we, however, also include at each vertex in Eq. (13) the form factor containing an arbitrary cutoff $\Lambda$

$$
F^{t}(\mathbf{k})=\left(\frac{2 \Lambda^{2}-M^{2}}{2 \Lambda^{2}+\mathbf{k}^{2}}\right)^{2}
$$

that was used in $[20,23]$ to obtain convergence in scattering calculations based on the relativistic $\hat{\Gamma}$ vertices. From Eq. (9) the coordinate space nonrelativistic $K \bar{K} \rightarrow K \bar{K}$ potential associated with the exchange of boson $M$ is then

$$
V(r)=-g^{2} C_{I} U(M, r)
$$

after division by $4 M_{K}^{2}$ where $M_{K}$ is the kaon mass, and $U(M, r)$ is the Fourier transform

$$
\begin{aligned}
U(M, r)= & \int \frac{d^{3} \mathbf{k}}{(2 \pi)^{3}} \frac{\left[F^{t}(\mathbf{k})\right]^{2}}{M^{2}+\mathbf{k}^{2}} e^{i \vec{k} \cdot \vec{r}} \\
= & \frac{1}{4 \pi}\left\{\frac{e^{-M r}}{r}-\frac{e^{-\sqrt{2} \Lambda}}{r}\left[1+\frac{1}{16}\left(11-\frac{M^{2}}{2 \Lambda^{2}}+\frac{M^{4}}{4 \Lambda^{4}}\right)\right.\right. \\
& \times\left(1-\frac{M^{2}}{2 \Lambda^{2}}\right)(\sqrt{2} \Lambda r) \\
& +\frac{1}{16}\left(3-\frac{M^{2}}{2 \Lambda^{2}}\right)\left(1-\frac{M^{2}}{2 \Lambda^{2}}\right)^{2}(\sqrt{2} \Lambda r)^{2} \\
& \left.\left.+\frac{1}{48}\left(1-\frac{M^{2}}{2 \Lambda^{2}}\right)^{3}(\sqrt{2} \Lambda r)^{3}\right]\right\} .
\end{aligned}
$$

The resulting $K \bar{K}$ potential due to $\rho, \omega, \phi$ exchange is attractive in both isospin channels $I=0$ and 1 ,

$$
\begin{aligned}
V_{I=0}(r)= & -g_{\rho \pi \pi}^{2}\left\{\frac{3}{4} U\left(M_{\rho}, r\right)+\frac{1}{4} U\left(M_{\omega}, r\right)\right. \\
& \left.+\frac{1}{2} U\left(M_{\phi}, r\right)\right\}
\end{aligned}
$$

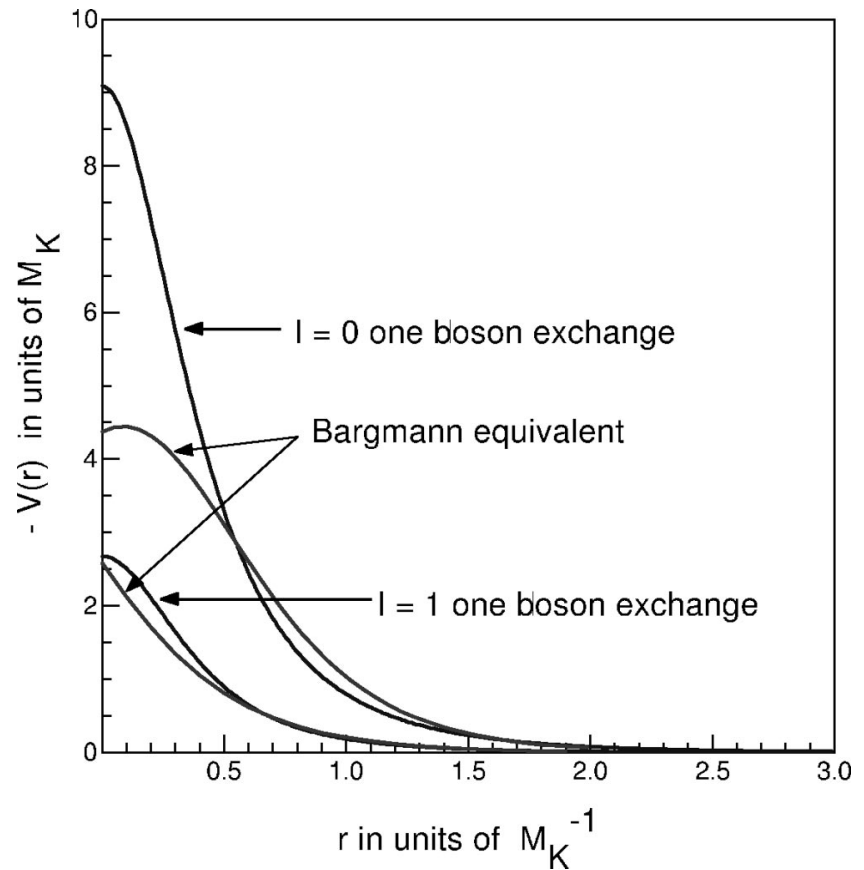

FIG. 1. One-boson exchange potentials using a cutoff of $\Lambda$ $=4 \mathrm{GeV}$. The coupling constant is $\alpha_{s}=2.9$ and the masses of the exchanged mesons are $\left(M_{\rho}, M_{\omega}, M_{\phi}\right)=(769,783,1019) \mathrm{MeV}$. The analytical Bargmann potentials that reproduce the same scattering lengths and effective ranges as the original one-boson exchange potentials are shown as companion curves.

and

$$
\begin{aligned}
V_{I=1}(r)= & -g_{\rho \pi \pi}^{2}\left\{-\frac{1}{4} U\left(M_{\rho}, r\right)\right. \\
& +\frac{1}{4} U\left(M_{\omega}, r\right) \\
& \left.+\frac{1}{2} U\left(M_{\phi}, r\right)\right\}
\end{aligned}
$$

but too weakly so in the latter channel due to an almost complete cancellation between $\rho$ and $\omega$ exchange to support a bound state. In deriving these expressions we have exploited the $S U(3)$ relations (12) to express all coupling constants in terms of $g_{\rho \pi \pi}$.

These potentials are plotted in Fig. 1 for a strong coupling constant $\alpha_{s}=g_{\rho \pi \pi}^{2} / 4 \pi=2.9$ and cutoff $\Lambda=4 \mathrm{GeV}$. In principle $\Lambda$ can be different for each exchanged boson [20]. One notes that the $V_{I}$ are now finite at the origin, in contrast to the sum of pure Yukawa potentials to which they reduce as $\Lambda$ $\rightarrow \infty$.

The coordinate space version of the wave equation Eq. (7) that describes the relative $s$-state motion in these potentials becomes the usual Schrödinger equation

$$
\left[\nabla^{2}+k^{2}-M_{K} V_{I}(r)\right] \psi(r)=0
$$

with total $\mathrm{CM}$ energy $P_{0}=2 M_{K}+k^{2} / M_{K}$. We ignore the charged to neutral kaon mass difference and work with an 


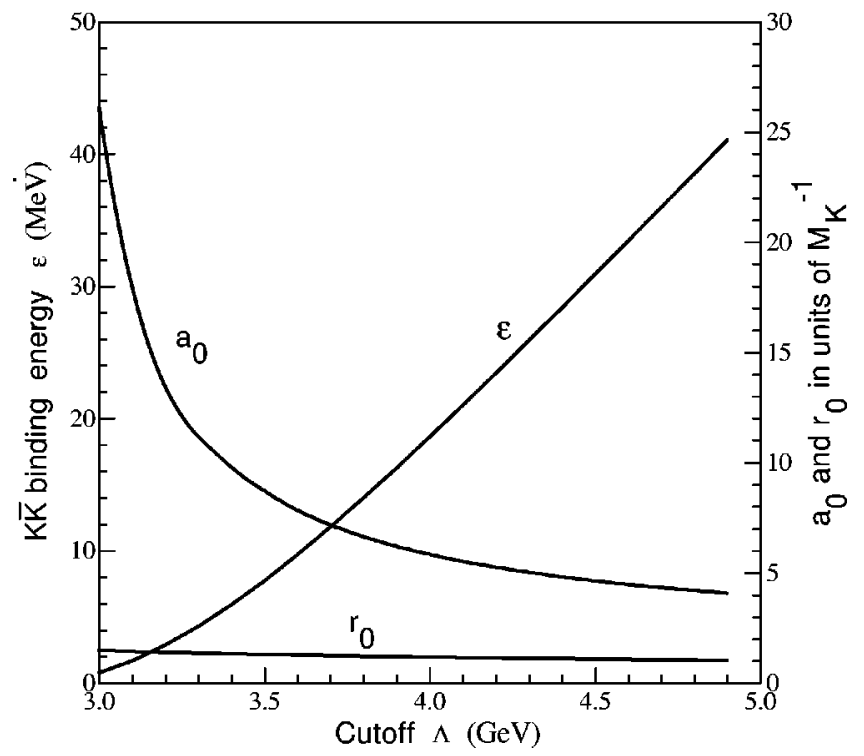

FIG. 2. Dependence of the $K \bar{K}$ binding energy $\varepsilon$ (left hand scale), scattering length $a_{0}$ and effective range $r_{0}$ (right hand scale) on the choice of cutoff $\Lambda$ used in the form factor for the isoscalar one-boson exchange potential.

average kaon mass $M_{K}$. By numerically constructing the $k$ $\rightarrow 0$ scattering solutions one easily obtains the scattering lengths and effective ranges that enter into the effective range expansion of the $s$-wave phase shifts $\delta(k)$

$$
k \cot \delta(k)=-\frac{1}{a_{I}}+\frac{1}{2} r_{I} k^{2}+\cdots
$$

in the two channels as

$$
\begin{aligned}
& a_{0}=5.835 M_{K}^{-1}, \quad r_{0}=1.187 M_{K}^{-1} \quad \text { (isoscalar) } \\
& a_{1}=-0.401 M_{K}^{-1}, \quad r_{1}=3.702 M_{K}^{-1} \quad \text { (isovector). }
\end{aligned}
$$

Thus only the isoscalar potential supports a bound state. Its binding energy is calculated to be $\varepsilon=18.63 \mathrm{MeV}$, placing the total mass for the bound $K \bar{K}$ system at $P_{0}=2 M_{K}-\varepsilon$ $=973.4 \mathrm{MeV}$. Figure 2 illustrates the sensitivity of $\varepsilon, a_{0}$ and $r_{0}$ on the choice of cutoff for this channel. For $\Lambda$ $\gtrsim 4 \mathrm{GeV}$ the values of $a_{0}$ and $r_{0}$ are only weakly dependent on $\Lambda$.

Even though the isovector potential does not support a bound state the $a_{0}(980)$ can be fully explained by this model as a threshold effect [21]. Effects of the $a_{0}(980)$ thus are included in the calculation though it should be noticed that decays to the $\pi \eta$-channel are not considered in the analytical model.

\section{B. Shape-independent approach}

The relatively small (on a hadronic scale) binding energy $\varepsilon / M_{K} \sim 0.04$ in the isoscalar channel (and no binding at all in the isovector channel) indicates that an effective range approach should be applicable. This is confirmed by recalculat- ing the binding energy from the effective range formula [25] $\varepsilon \approx \kappa^{2} / M_{K}=18.60 \mathrm{MeV}$ where $\kappa=-a=0.194 M_{K}$, see Eq. (27) below. Consequently it is entirely sufficient to characterize the $K \bar{K}$ interaction at low energies in terms of a scattering length and an effective range: the actual shape of the potential is immaterial.

We make use of this feature in the following to replace the strong potentials $V_{I}$ in Eqs. (17) and (18) by analytic potentials of the Bargmann type given below that are phase equivalent to them. Bargmann [24] (see also [34] for an in depth overview) has shown how to construct families of potentials that give rise to a prescribed Jost function $f(k)$. If one chooses

$$
f(k)=\frac{k-i a}{k-i b}
$$

then the potential that leads to this Jost function can be determined. It is $[24,34,35]$,

$$
V_{I}(r)=-\frac{1}{M_{K}} \frac{8 b^{2}}{b^{2}-a^{2}}\left[\frac{e^{b r}}{b-a}+\frac{e^{-b r}}{b+a}\right]^{-2} .
$$

For this potential the effective range expansion is exact:

$$
k \cot \delta(k)=\frac{b a}{b-a}+\frac{k^{2}}{b-a} .
$$

This allows one to identify the scattering length and effective range as

$$
a_{I}=-\frac{b-a}{b a}, \quad r_{I}=\frac{2}{b-a}
$$

where the actual values of the parameters $a$ and $b$ will depend on the isospin channel.

Fixing $a$ and $b$ is obviously equivalent to prescribing the scattering length and effective range. If $a$ is negative, $a$ $=-\kappa$, say, then $f(k)$ has a zero at $k=-i \kappa$ in the lower half of the complex $k$ plane, and there is a bound state [34] of binding energy $\varepsilon_{s}=\kappa^{2} / M_{K}$. Both the scattering and bound state wave functions of the Bargmann potential in Eq. (23) are known explicitly. Further details will be found in the Appendix. The availability of such analytic solutions will be important for including the effects of the $K \bar{K} \rightarrow \pi \pi$ decay channel nonperturbatively as discussed below.

Now invert Eq. (25) to construct phase equivalent potentials that have the same scattering length and effective range as the original exchange potentials in Eqs. (17) and (18). The required values of $a$ and $b$ are

$$
\begin{aligned}
& a=-\frac{1}{r_{I}}\left[1-\sqrt{1-2 r_{I} / a_{I}}\right] \\
& b=\frac{1}{r_{I}}\left[1+\sqrt{1-2 r_{I} / a_{I}}\right]
\end{aligned}
$$


TABLE I. Parameters of the Bargmann potentials, Eq. (23), that give rise to identical scattering lengths and effective ranges as the one boson exchange potentials in Eqs. (17) and (18). The last column gives the value of the logarithmic derivative that determines the strength of the annihilation potential. The units are $M_{K}$.

\begin{tabular}{lccc}
\hline \hline Isospin & $a$ & $b$ & $\zeta$ \\
\hline$I=0$ & -0.1936 & 1.491 & $-17.409 i$ \\
$I=1$ & +0.9219 & 1.462 & $-26.114 i$ \\
\hline \hline
\end{tabular}

$$
\begin{array}{lll}
a=-0.1936 \mathrm{M}_{\mathrm{K}} & b=1.491 \mathrm{M}_{\mathrm{K}} \quad \text { (isoscalar) } \\
a=+0.9219 \mathrm{M}_{\mathrm{K}} & b=1.462 \mathrm{M}_{\mathrm{K}} \quad \text { (isovector) }
\end{array}
$$

for the two channels in question. These parameters are summarized in Table I for easy reference. The resulting set of phase equivalent potentials are included in Fig. 1.

\section{C. $K \bar{K}$ annihilation potential}

From Eq. (13) one reads off the irreducible vertex for $K \bar{K} \rightarrow \pi \pi$ via $K^{*}$ exchange, as

$$
\hat{\Gamma}(K \bar{K} \rightarrow \pi \pi) \approx-C_{I} n_{s} g_{\pi K K^{*}}^{2} \frac{\left(M_{\pi}+M_{K}\right)^{2}}{M_{K^{*}}^{2}}
$$

where $n_{S} C_{I}=-\sqrt{3}$ or $-\sqrt{2}$ in the isoscalar or isovector channel. We have neglected the 3-momentum transfer relative to the large $K^{*}(892)$ mass. As shown in Fig. 3, this amounts to introducing point vertices that can contribute to $K \bar{K}$ scattering via an intermediate pion loop. The contribution from this interaction is complex due to the possibility of on-shell decays $K \bar{K} \rightarrow \pi \pi$ in the intermediate state, and the resulting $K \bar{K}$ bound state acquires a width. The details are as follows: calling the contribution from Fig. $3 i \hat{\Gamma}^{(1)}$ one finds

$$
\begin{aligned}
i \hat{\Gamma}^{(1)}= & i \hat{\Gamma}^{2}(K \bar{K} \rightarrow \pi \pi) \\
& \times \int \frac{d^{3} \mathbf{l}}{(2 \pi)^{3}} \int_{-\infty}^{\infty} \frac{d l_{0}}{2 \pi i} D\left(l+\frac{1}{2} P_{0}\right) D\left(-l+\frac{1}{2} P_{0}\right) \\
= & i \hat{\Gamma}^{2}(K \bar{K} \rightarrow \pi \pi) \frac{1}{4 M_{\pi}^{2}} \int \frac{d^{3} \mathbf{l}}{(2 \pi)^{3}} \frac{1}{\mathbf{l}^{2} / M_{\pi}+2 M_{\pi}-P_{0}} \\
= & i \hat{\Gamma}^{2}(K \bar{K} \rightarrow \pi \pi) \frac{J\left(P_{0}\right)}{4 M_{\pi}^{2}}
\end{aligned}
$$

in view of Eq. (8). As summarized briefly in Eqs. (A23) and (A24) of the Appendix the three dimensional integral $J\left(P_{0}\right)$ can be dimensionally regulated in $d$ dimensions without difficulty to obtain a finite result [16] for $d \rightarrow 3$. The associated annihilation potential $V_{K \bar{K}}$ is then obtained from Eq. (9) as an attractive contact potential in coordinate space,

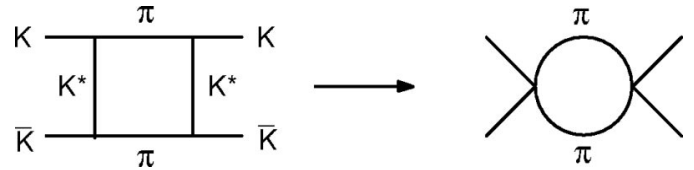

FIG. 3. Replacement of the irreducible $K \bar{K}$ scattering diagram involving the exchange of two $K^{*}$ 's by an equivalent pion loop diagram with point vertices.

$$
V_{K \bar{K}}(r)=-\frac{\hat{\Gamma}^{2}(K \bar{K} \rightarrow \pi \pi)}{\left(4 M_{K}^{2}\right)\left(4 M_{\pi}^{2}\right)} J\left(P_{0}\right) \delta^{3}(\mathbf{r})=-c_{0}^{2} \delta^{3}(\mathbf{r})
$$

with

$$
c_{0}^{2}=\frac{3}{16} \frac{\left(g_{\pi K K^{*}}^{2}\right)^{2}}{M_{\pi}^{2} M_{K}^{2}}\left(\frac{M_{\pi}+M_{K}}{M_{K^{*}}}\right)^{4} J\left(P_{0}\right) \quad \text { (isoscalar). }
$$

The value of this factor in the isovector channel is $c_{1}^{2}$ $=\frac{2}{3} c_{0}^{2}$. The function

$$
J\left(P_{0}\right)=i \frac{M_{\pi}}{4 \pi}\left[M_{\pi}\left(P_{0}-2 M_{\pi}\right)\right]^{1 / 2}
$$

is a complex function of $P_{0}$ that is positive and pure imaginary along the upper lip of the branch cut extending from $2 M_{\pi}$ to $\infty$ along the real $P_{0}$ axis, see Eq. (A24).

\section{Jost function for a finite plus delta function potential at the origin}

We investigate the influence of the annihilation potential on the properties of the $K \bar{K}$ system by constructing the revised Jost function for the sum $V_{I}+V_{K \bar{K}}$. Since $V_{K \bar{K}}$ is a contact interaction, and the solutions in the potential $V_{I}$ are known, this can be done without approximation. The new scattering problem to be solved reads

$$
\left[\nabla^{2}+k^{2}-M_{K} V_{I}(r)+M_{K} c_{I}^{2} \delta^{3}(\mathbf{r})\right] \psi(r)=0 .
$$

Consider isospin $I=0$. The presence of the delta function potential obliges one to give up the boundary condition $\varphi(k, r) \sim r \rightarrow 0$ on the scattering wave function $\varphi(k, r)$ $=r \psi(k, r)$ at the origin in favor of prescribing [36] its logarithmic derivative $\varphi^{\prime} / \varphi$ there [34]. By integrating Eq. (33) over the volume of an infinitesimal sphere centered at the origin, one finds that the logarithmic derivative at the origin is fixed by the strength of the delta potential according to

$$
\frac{\varphi^{\prime}}{\varphi}=\frac{4 \pi}{M_{K} c_{0}^{2}}=\zeta
$$

The point is now that for $r>0$ both the boundary condition on $\varphi(k, r)$ and the closed form of irregular solution of Eq. (33) are known explicitly: they are given by Eqs. (34) and (A4) respectively. By constructing the Wronskian of this pair in the limit $r \rightarrow 0$ one obtains the revised Jost function as 


$$
f(k)=W[f, \varphi]=\left[(\zeta+i k) \frac{k-i a}{k-i b}+i \frac{b^{2}-a^{2}}{k-i b}\right]
$$

after setting $\lim \varphi(k, r) \rightarrow 1$ for convenience. This choice is unimportant since neither the root of $f(k)$ nor the scattering phase shift depends on it. The value of the logarithmic derivative $\zeta$ is $k$-dependent through $P_{0}$ : from Eqs. (31) and (34) one finds

$$
\begin{aligned}
\zeta(k) & =-\frac{256 i}{3 \alpha_{s}^{2}} M_{\pi} M_{K}\left(\frac{M_{K^{*}}}{M_{K}+M_{\pi}}\right)^{4}\left[M_{\pi}\left(P_{0}-2 M_{\pi}\right)\right]^{-1 / 2} \\
P_{0} & =2 M_{K}+\frac{k^{2}}{M_{K}}
\end{aligned}
$$

after taking the $S U(3)$ value $g_{\pi K K^{*}}=-g_{\rho \pi \pi} / 2$ for the coupling constant.

\section{NUMERICAL RESULTS}

The single equation (35) contains all the necessary information on the scattering and bound states of the two-body $K \bar{K}$ system. Moreover, this information can be extracted without further approximation. We use this form of $f(k)$ to identify the revised scattering length and effective range as well as the bound state energy in the presence of $K \bar{K} \rightarrow 2 \pi$ annihilation.

\section{A. Effective range expansion}

The $K \bar{K} \rightarrow \pi \pi$ annihilation channel renders the scattering matrix $S(k)=f(k) / f(-k)$ nonunitary, since now $f^{*}(k)$ $\neq f(-k)$. This can be seen directly from Eq. (35) after noting that the logarithmic derivative $\zeta$ is even in $k$ and purely negative imaginary for real $k$. The scattering phase shift is now complex. In order to identify the scattering length and effective range in the presence of absorption, one can either maintain the expansion (20), thereby introducing complex effective range parameters [37,31], or define [38] these in terms of the real part $\delta(k)$ of the total phase shift [not the phase of $S(k)]$,

$$
S(k)=f(k) / f(-k)=\eta e^{2 i \delta(k)}, \quad 0<\eta<1 .
$$

In the latter case

$$
k \cot \delta(k)=i k \frac{S(k)+\eta}{S(k)-\eta}=i k \frac{f(k)|f(-k)|+f(-k)|f(k)|}{f(k)|f(-k)|-f(-k)|f(k)|}
$$

that shows explicitly that $k \cot \delta(k)$ is an even function of $k$. Set $\zeta(k)=-i \xi(k)$ in Eq. (35) for the Jost function, and then expand the right hand side to $O\left(k^{2}\right)$. After some calculation one establishes the revised effective range expansion

$$
k \cot \delta(k)=-\frac{1}{a_{0}^{\prime}}+\frac{1}{2} r_{0}^{\prime} k^{2}+\cdots
$$

with the exact expressions

$$
-\frac{1}{a_{0}^{\prime}}=\frac{b}{b-a}\left\{\frac{a^{2} \xi^{2}+\left(b^{2}-a^{2}\right)^{2}}{a \xi^{2}-2 a b^{2}+a^{3}-b^{3}}\right\}
$$

and

$$
\begin{aligned}
\frac{1}{2} r_{0}^{\prime}= & \frac{a^{2}\left(b^{2}-a^{2}\right)^{4}+a(b+a)\left[a^{3}(b-a)\left(5 b^{2}-3 a^{2}\right)+b^{4}\left(a^{2}-2 a b+2 b^{2}\right)\right] \xi^{2}}{(b-a)\left[a^{2} \xi^{2}+\left(b^{2}-a^{2}\right)^{2}\right]\left[a^{3}-b^{3}-2 a b^{2}+a \xi^{2}\right]^{2}} \\
& +\frac{a(b+a)\left[a(b-a)\left(b^{2}-3 a^{2}\right)-b^{4}\right] \xi^{4}+a^{4} \xi^{6}}{(b-a)\left[a^{2} \xi^{2}+\left(b^{2}-a^{2}\right)^{2}\right]\left[a^{3}-b^{3}-2 a b^{2}+a \xi^{2}\right]^{2}}-2 \xi \xi^{\prime} a b^{4} \frac{\left[\left(a^{2}+a b-b^{2}\right)\left(a^{3}-a b^{2}-b^{3}\right)+a^{2} \xi^{2}(b+a)\right]}{(b-a)\left[a^{2} \xi^{2}+\left(b^{2}-a^{2}\right)^{2}\right]\left[a^{3}-b^{3}-2 a b^{2}+a \xi^{2}\right]^{2}}
\end{aligned}
$$

for the new inverse scattering length and effective range. Here

$$
\begin{aligned}
\xi & =i \zeta=\frac{256}{3 \alpha_{s}^{2}} M_{\pi} M_{K}\left(\frac{M_{K^{*}}}{M_{K}+M_{\pi}}\right)^{4}\left[M_{\pi}\left(2 M_{K}-2 M_{\pi}\right)\right]^{-1 / 2} \\
& =17.409 M_{K} \\
\xi^{\prime} & =i \frac{\partial \zeta}{\partial k^{2}}=-\frac{128}{3 \alpha_{s}^{2}} \sqrt{M_{\pi}}\left(\frac{M_{K^{*}}}{M_{K}+M_{\pi}}\right)^{4}\left[\left(2 M_{K}-2 M_{\pi}\right)\right]^{-3 / 2} \\
& =-6.064 M_{K}^{-1}
\end{aligned}
$$

at $k=0$, and the primes serve to distinguish these scattering lengths and ranges from those appearing in Eq. (24), to which they reduce in the no annihilation limit $\xi \rightarrow \infty$. The numerical values of $\xi$ and $\xi^{\prime}$ have been calculated for meson masses $M_{\pi}=140 \mathrm{MeV}, M_{K}=496 \mathrm{MeV}, M_{K^{*}}=892 \mathrm{MeV}$ and coupling constant $\alpha_{s}=2.9$. Using these together with the values for $a$ and $b$ given in Table I, one obtains

$$
a_{0}^{\prime}=4.281 M_{K}^{-1} ; \quad r_{0}^{\prime}=3.203 M_{K}^{-1} \quad \text { (isoscalar) }
$$

as predictions for the $K \bar{K}$ isoscalar scattering length and effective range in the presence of absorption. The corresponding values for the isovector channel are still 
TABLE II. Summary of calculated isoscalar $K \bar{K}$ scattering lengths $a_{0}^{\prime}$ and effective ranges $r_{0}^{\prime}$ (in units $M_{K}^{-1}$ ), based on the $a$ and $b$ Bargmann potential parameters (in units $M_{K}$ ) that reproduce the complex bound state at $P_{0}$ shown in column two. The physical constants that enter into these calculations are $\left(M_{\pi}, M_{K}, M_{K^{*}}\right)=(140,496,892) \mathrm{MeV}$, and $\alpha_{s}=g_{\rho \pi \pi^{2}}^{2} / 4 \pi \approx 2.9$, using the KSRF value of $g_{\rho \pi \pi} \approx 6$. The last four rows list the values of $a$ and $b$, and the resulting isoscalar scattering lengths and effective ranges that would be required to reproduce the theoretical results of Weinstein and Isgur (WI), the fits of Morgan and Pennington (MP), and the experimental data.

\begin{tabular}{lccccc}
\hline \hline \multicolumn{1}{c}{ Source } & $P_{0}=M-i \Gamma / 2(\mathrm{MeV})$ & $a$ & $b$ & $a_{0}^{\prime}$ & $r_{0}^{\prime}$ \\
\hline This calculation & $981-25 i$ & -0.194 & 1.491 & 4.28 & 3.20 \\
WI [6] & $972-16 i$ & -0.213 & 1.170 & 5.01 & 2.12 \\
MP [40] & $970-24 i$ & -0.231 & 1.367 & 4.30 & 2.16 \\
PDG [42] & $(974 \pm 3)-(24 \pm 5) i$ & $-0.22 \pm 0.02$ & $1.41 \pm 0.16$ & $4.35 \pm 0.49$ & $2.46 \pm 0.35$ \\
E791 [26] & $(975 \pm 3)-(22 \pm 2) i$ & $-0.21 \pm 0.02$ & $1.37 \pm 0.05$ & $4.52 \pm 0.20$ & $2.51 \pm 0.33$ \\
\hline \hline
\end{tabular}

$$
a_{1}^{\prime}=-0.401 M_{K}^{-1} ; \quad r_{1}^{\prime}=3.702 M_{K}^{-1} \quad \text { (isovector) }
$$

since there is no annihilation contribution from the $l=0, I$ $=1$ partial wave to the $K \bar{K}$ scattering amplitude due to the identity of the outgoing pions in the isospin representation. Higher partial waves cannot contribute in any event due to the contact nature of the annihilation interaction. In fact the $a_{0}(980) \rightarrow 2 \pi$ decay channel is forbidden by $G$ parity. Thus the isovector scattering length remains real if, as here, the $\pi \eta$ decays are ignored.

\section{B. Bound states for $I=0$}

The bound states of Eq. (33) are determined [34] by the complex $\operatorname{root}(\mathrm{s})$ of $f(k)$ in Eq. (35), with $\operatorname{Im} k<0$, i.e.

$$
[\zeta(k)+i k](k-i a)+i\left(b^{2}-a^{2}\right)=0 .
$$

Let $k=p-i \kappa, \kappa>0$ be such a root. Then the total mass $M$ and decay width $\Gamma$ of a bound $K \bar{K}$ pair at rest reads

$$
P_{0}=M-\frac{i}{2} \Gamma=2 M_{k}+\frac{(p-i \kappa)^{2}}{M_{K}} .
$$

This root can only be found numerically as will be done shortly [39]. However, since the magnitude of the absorptive coupling $M_{K}^{2}\left|c_{0}^{2}\right| \sim 4 \pi M_{K} / \xi \sim 0.7$ is quite small relative to the isoscalar potential strength, a perturbative solution in inverse powers of $\zeta$ is useful. Replace $\zeta$ by its value $-i \xi$ at $k=0$ (more correctly $k \approx-i \kappa$ but the difference is quantitatively insignificant). Then Eq. (45) reduces to a quadratic equation for $k$. The relevant root is

$$
k=\frac{b^{2}-\kappa^{2}}{\xi}-i \kappa+O\left(\xi^{-2}\right) \approx(0.1255-0.1936 i) M_{K}
$$

where $k=i a=-i \kappa=-0.1936 M_{K} i$ is the root leading to the bound state in the absence of absorption, and $b$ $=1.491 M_{K}$. Working to the same order in $\xi^{-1}$ (i.e. neglecting the real part of $k$ ), one finds that

$$
P_{0} \approx 2 M_{K}-\frac{\kappa^{2}}{M_{K}}-2 i \kappa \frac{\left(b^{2}-\kappa^{2}\right)}{M_{K} \xi} .
$$

Since $4 \pi \psi^{2}(0)=2 \kappa\left(b^{2}-\kappa^{2}\right)$ from Eq. (A10) the result in Eq. (48) is equivalent to treating the annihilation potential $-c_{0}^{2} \delta^{3}(\mathbf{r})$ as a first order perturbation of $P_{0}$,

$$
P_{0} \approx 2 M_{K}-\frac{\kappa^{2}}{M_{K}}-\frac{i}{2} \Gamma ; \quad \Gamma=-2 i c_{0}^{2} \psi^{2}(0)=\frac{8 \pi}{M_{K} \xi} \psi^{2}(0)
$$

Upon recalling that $-i \xi^{-1} \sim-J\left(P_{0}\right)$, one sees from this form that the complex solution for $P_{0}$ always lies on the second sheet $-2 \pi \leqslant \phi \leqslant 0$ of the cut complex $P_{0}$ plane for $J\left(P_{0}\right)$. Otherwise the signs of the imaginary parts of $P_{0}$ and $-J\left(P_{0}\right)$ will differ, and no solution is possible. Taking cognizance of this fact the exact root of Eq. (45) and the resulting value for $P_{0}$ are found numerically to be

$$
k=(0.1254-0.1972 i) M_{K} ; \quad P_{0}=(981-25 i) \mathrm{MeV}
$$

using the previously determined values of $a$ and $b$ shown in Table I. Notice that the exact value of $k$ lies rather close to the perturbation estimate given by Eq. (47).

The exact numerical results for the Bargmann potential that is phase-equivalent to Eq. (17) are given in the first row of Table II. We contrast these results with the mass and width calculations based on the Weinstein-Isgur nonrelativistic potential model [6], as well as the predictions of Morgan and Pennington [40], based on a parametrization of the Jost function [41] with parameters determined from experimental phase shifts. These calculations are also compared with the earlier experimental results summarized by the Particle Data Group (PDG) [42], as well as the recent data from the Fermilab E791 Collaboration [26].

In order to extract Bargmann potential parameters that would lead to the mass and width values of either WeinsteinIsgur, or Morgan and Pennington, we have turned Eq. (45) around and asked what values of $a$ and $b$ produce a root $k$ $=p-i \kappa$ that gives a prescribed value of the complex total energy in Eq. (46). The answer is 


$$
\begin{aligned}
& a=-\kappa-p \frac{\nu+\kappa}{p-\xi} \\
& b=\sqrt{a^{2}-p(p-\xi)+(\nu+\kappa)(a+\kappa)}
\end{aligned}
$$

after setting $\zeta(p-i \kappa)=\nu-i \xi$. We have treated the experimental data in the same way. These relations allow one to establish a direct link between the mass and decay width of the bound $K \bar{K}$ state, and thence extract the isoscalar scattering length and effective range via Eqs. (40) and (41), or Eqs. (63) and (64).

The effective range expansion parameters obtained in this way for the Weinstein-Isgur calculations, the MorganPennington fits, the PDG data, and the Fermilab data, are also shown in Table II. Collectively these results are all internally consistent, and suggest that the nonrelativistic OBE potential that includes absorption via $K^{*}$ exchange, provides a natural description for the properties of the $f_{0}(980)$ scalar meson as a bound $K \bar{K}$ pair. In particular, the prediction of the correct decay width from $K^{*}$ exchange, which is parameterfree and can be treated without approximation, adds weight to this interpretation. In this connection it is also interesting to compare with the current-algebra prediction [43] of $\Gamma$ $\approx 660 \mathrm{MeV}$ for the much larger decay width (600-1000 $\mathrm{MeV}$, also dominantly into two pions) of the $f_{0}(400$ $-1200)$ or $\sigma$ scalar meson, that is considered as a candidate for the $q \bar{q}$ bound state.

\section{Two-photon decay of $f_{0}(980)$}

The two-photon decay of the $f_{0}(980)$, considered as a bound kaon-antikaon pair, can be treated by the same method. Working in a suitable gauge, the two-photon annihilation potential $V_{\gamma \gamma}$ is given by the single diagram in Fig. 3 with the $K \bar{K} \rightarrow 2 \pi$ vertices and pion propagators replaced by the analagous electromagnetic $K^{+} K^{-} \rightarrow 2 \gamma$ ("seagull") vertices and photon propagators, after division by two to compensate for crossing. One obtains

$$
V_{\gamma \gamma}=-c_{\gamma \gamma}^{2} \delta^{3}(\mathbf{r})
$$

Without counterterms, $c_{\gamma \gamma}^{2}$ is formally divergent due to the photon loop. However, the imaginary part is finite,

$$
\operatorname{Im} c_{\gamma \gamma}^{2}=\frac{1}{2}\left(\frac{2 \pi \alpha^{2}}{M_{K}^{2}}\right)
$$

where $\alpha=e^{2} / 4 \pi \approx 1 / 137$ is the fine structure constant. The two-photon decay width then follows from the second expression in Eq. (49) as

$$
\begin{aligned}
\Gamma_{\gamma \gamma} & =2 \operatorname{Im} c_{\gamma \gamma}^{2} \psi_{K^{+} K^{-}}^{2}=\frac{2 \pi \alpha^{2}}{M_{K}^{2}} \psi_{K^{+} K^{-}}^{2} \\
& =\frac{\alpha^{2} \kappa\left(b^{2}-\kappa^{2}\right)}{2 M_{K}^{2}}=5.59 \mathrm{keV}
\end{aligned}
$$

where $\psi_{K^{+} K^{-}}=\psi\left\langle K^{+} K^{-} \mid K \bar{K}\right\rangle$ is the probability amplitude for finding a $K^{+} K^{-}$pair in the $f_{0}(980)$ ground state. For a state of good isospin, $\left\langle K^{+} K^{-} \mid K \bar{K}\right\rangle=1 / \sqrt{2}$. One can verify this result independently by noting that the coefficient $2 \pi \alpha^{2} / M_{K}^{2}=v \sigma_{\gamma \gamma}$, where $\sigma_{\gamma \gamma}$ gives the low velocity cross section for the annihilation of free, charged boson-antiboson pairs of relative velocity $v$ into two photons [44]. The decay width of the bound state is then calculated in the standard way [44] as $\sigma_{\gamma \gamma}$ times the flux density, or $\sigma_{\gamma \gamma} \times v \psi_{K^{+} K^{-}}^{2}$, which just reproduces Eq. (55).

The numerical value found above for $\Gamma_{\gamma \gamma}$ is an order of magnitude larger than both the estimate given in [45] and also the currently quoted value of $0.39_{-0.13}^{+0.10} \mathrm{keV}$ from experiment [46]. In the molecular picture this discrepancy comes about because of the extreme sensitivity of the spatial wave function at the origin to the assumed binding energy of the $K \bar{K}$ pair. In [45] the author estimates $\psi(0) \approx \frac{1}{10} M_{K}^{3 / 2}$ using a Gaussian potential fitted to a binding energy of $10 \mathrm{MeV}$. This is a factor $\sim 3$ smaller than our estimate of $\psi(0)$ that corresponds to a binding energy of $18.6 \mathrm{MeV}$, which in turn increases our estimated width by an order of magnitude over that given in [45] (see Fig. 9).

On the other hand the $2 \pi$ decay width, which is also proportional to $\psi^{2}(0)$, is correctly reproduced by our wave function. Thus it would not seem possible to reproduce both the measured pole position of the $f_{0}(980)$ decaying state in the complex plane and at the same time the two photon decay width in the molecular picture without introducing some additional mechanism that reduces the fractional parentage $f_{p}=\left\langle K^{+} K^{-} \mid K \bar{K}\right\rangle$ of $K^{+} K^{-}$in the $f_{0}(980)$ ground state. For, apart from this factor, the branching ratio of electromagnetic to hadronic decays is given by the expression

$$
\begin{aligned}
\frac{\Gamma_{\gamma \gamma}}{\Gamma} & \approx \frac{\alpha^{2} \xi}{8 M_{K}}\left|f_{p}\right|^{2} \\
& =\frac{2}{3 \sqrt{2}}\left(\frac{e^{2}}{g_{\pi K K^{*}}^{2}}\right)^{2}\left(\frac{M_{K^{*}}}{M_{\pi}+M_{K}}\right)^{4}\left(\frac{M_{\pi}}{M_{K}-M_{\pi}}\right)^{1 / 2}\left|f_{p}\right|^{2} \\
& \approx 1.2 \times 10^{-4}\left|f_{p}\right|^{2}
\end{aligned}
$$

that contains known physical constants if one adopts the $S U(3)$ value $g_{\pi K K^{*}}=-1 / 2 g_{\rho \pi \pi}$ for the $K \rightarrow \pi K^{*}$ coupling constant again. The only less well-determined constant in this expression is $g_{\rho \pi \pi}$. However, the spread between the various estimates $[20,21]$ of this coupling is not nearly large enough to cause the branching ratio to alter by an order of magnitude. This then leaves $f_{p}$. Since $\Gamma_{\gamma \gamma} / \Gamma \sim 10^{-5}$ experimentally, one would require $f_{p} \sim 1 / \sqrt{10}$ down from $1 / \sqrt{2}$, suggesting that the assumption of a pure $K \bar{K}$ molecular ground state of good isospin is oversimplified.

A competing picture for the structure of $f_{0}(980)$ as a fourquark state has been developed in [5], which leads to an estimate $\Gamma\left(f_{0}(980) \rightarrow \gamma \gamma\right) \approx 0.27 \mathrm{keV}$ [47] that is also (with a particular choice of parameters) not in contradiction with experiment. One has therefore to conclude that agreement or otherwise between the calculated and experimental $2 \gamma$ decay 
TABLE III. Complex scattering lengths and effective ranges for isospin zero and one scattering channels as calculated from Eq. (63), as well as from coupled channel calculations with the $f_{0}^{\prime}$ and the $\pi \eta$-channel [30] included (in units of $M_{K}^{-1}$ ).

\begin{tabular}{lccc}
\hline \hline & Isospin & $a_{I}^{\text {eff }}$ & $r_{I}^{\text {eff }}$ \\
\hline One-boson exchange & $\mathrm{I}=0$ & $4.281-2.398 \mathrm{i}$ & $1.169-0.178 \mathrm{i}$ \\
& $\mathrm{I}=1$ & -0.401 & 3.702 \\
$+f_{0}^{\prime}$ mixing $+\pi \eta$-channel & $\mathrm{I}=0$ & $2.165-4.592 i$ & $0.151-0.404 i$ \\
& $\mathrm{I}=1$ & $0.147-1.944 i$ & $-1.463-0.202 i$ \\
\hline \hline
\end{tabular}

width of $f_{0}(980)$ by itself is too ambiguous for deciding between a molecular versus a $q^{2} \bar{q}^{2}$ four-quark structure of this meson, and that some mixture of these two extreme pictures is probably indicated.

\section{D. $K \bar{K}$ cross sections and complex scattering lengths}

The linear combination of scattering amplitudes of isospin zero and one, $f_{p}=\left(f_{0}+f_{1}\right) / 2=\left(S_{p}-1\right) / 2 i k$ determines the $s$-wave $S$ matrix as $S_{p}=\left(S_{0}+S_{1}\right) / 2$ for the scattering and reaction cross sections for $K \bar{K}$ scattering in the absence of Coulomb interactions. One has

$$
\sigma_{\text {elas }}=\frac{\pi}{k^{2}}\left|1-S_{p}\right|^{2}, \quad \sigma_{r}=\frac{\pi}{k^{2}}\left(1-\left|S_{p}\right|^{2}\right)
$$

where $k$ is the relative momentum of the colliding pair. The reaction cross section has in turn contributions from the (quasielastic) "charge transfer" channel, $K^{+} K^{-} \leftrightarrow K^{0} \bar{K}^{0}$, in addition to the two-pion annihilation channel. One finds $\sigma_{r}$ $=\sigma_{e x}+\sigma_{a}$, with

$$
\sigma_{e x}=\frac{\pi}{k^{2}}\left|\left(\frac{S_{0}-S_{1}}{2}\right)\right|^{2}, \quad \sigma_{a}=\frac{1}{2}\left(\sigma_{a}^{(0)}+\sigma_{a}^{(1)}\right)
$$

since the annihilation cross section $\sigma_{a}$ is simply the average of the corresponding annihilation cross sections

$$
\sigma_{a}^{(I)}=\frac{\pi}{k^{2}}\left(1-\left|S_{I}\right|^{2}\right)
$$

in the separate isospin channels that add incoherently.

$S$ matrix elements of good isospin, $S_{I}$, appearing in these expressions are given directly by Eq. (37) in terms of the Jost functions for each isospin channel as

$$
S_{I}(k)=\left[\left(\frac{(\xi-k)(k-i a)-\left(b^{2}-a^{2}\right)}{(\xi+k)(k+i a)+\left(b^{2}-a^{2}\right)}\right)\left(\frac{k+i b}{k-i b}\right)\right]_{I}
$$

where the parameters in this expression refer to a specific isospin as indicated. Then

$$
\sigma_{\text {elas }}^{(I)}=\frac{4 \pi}{b^{2}+k^{2}}\left[\frac{\left(b^{2}-a^{2}+b a+k^{2}\right)^{2}+(b-a)^{2} \xi^{2}}{a^{2}(\xi+k)^{2}+\left[b^{2}-a^{2}+k(\xi+k)\right]^{2}}\right]
$$

$$
\sigma_{a}^{(I)}=\frac{4 \pi \xi}{k}\left[\frac{b^{2}+k^{2}}{a^{2}(\xi+k)^{2}+\left[b^{2}-a^{2}+k(\xi+k)\right]^{2}}\right] .
$$

Note that $\sigma_{a}^{(I)} \sim 1 / k$ at small $k$ as befits a true absorption cross section. One can confirm the expression for the annihilation cross section $\sigma_{a}^{(I)}$ by considering the flux loss in each isospin channel out of the radial wave function $\varphi(k, r) / f(-k)$ for scattering, into an infinitesimal sphere located at the origin. Employing the boundary conditions $\varphi(k, r) \rightarrow 1, \varphi^{\prime}(k, r)$ $\rightarrow \zeta$ as $r \rightarrow 0$ used to obtain the Jost function $f(k)$ of Eq. (35), this flux loss is given without approximation by $8 \pi /\left[M_{K}|f(-k)|^{2}\right]$. This is identical with the right hand side of the second of Eqs. (61) after division by the incident flux $v=2 k / M_{K}$.

Finally, taking the first option mentioned above Eq. (37) and expanding $k \cot \delta_{I}(k)$, where $\delta_{I}$ is the phase of $S_{I}(k)$ $=\exp \left(2 i \delta_{I}\right)$, one has

$$
k \cot \delta_{I}(k)=-1 / a_{I}^{e f f}+\frac{1}{2} r_{I}^{e f f} k^{2}+\cdots
$$

where the complex scattering length and effective range are given by the much simpler expressions

$$
a_{I}^{e f f}=\left[\frac{b^{2}-a^{2}+b a-i(b-a) \xi}{b\left(b^{2}-a^{2}+i a \xi\right)}\right]
$$

and

$$
r_{I}^{e f f}=-\frac{2(b-a)(\xi+i a)^{2}}{\left.\left[b^{2}-a^{2}+b a-i(b-a) \xi\right)\right]^{2}} .
$$

Values of these quantities in the $I=0$ and $I=1$ channels are listed in Table III. Note that for $I=1$, these expressions reduce to those in Eq. (25). The $\operatorname{Re}\left(a_{I}^{\text {eff }}\right)$ of course coincide with the previous results, Eq. (40), for the scattering lengths when annihilation is present. From Eqs. (57) to (59) the zero momentum elastic, charge transfer, and annihilation cross sections are given by

$$
\sigma_{\text {elas }}=4 \pi\left|a_{p}^{\text {eff }}\right|^{2}, \quad \sigma_{\text {ex }}=4 \pi\left|a_{n}^{e f f}\right|^{2}, \quad \sigma_{a}=-\frac{4 \pi}{k} \operatorname{Im}\left(a_{p}^{\text {eff }}\right)
$$

in the effective range approximation in terms of $a_{p}^{e f f}=\left(a_{0}^{e f f}\right.$ $\left.+a_{1}^{e f f}\right) / 2$, the common $K^{+} K^{-}$or $K^{0} \bar{K}^{0}$ scattering length in 


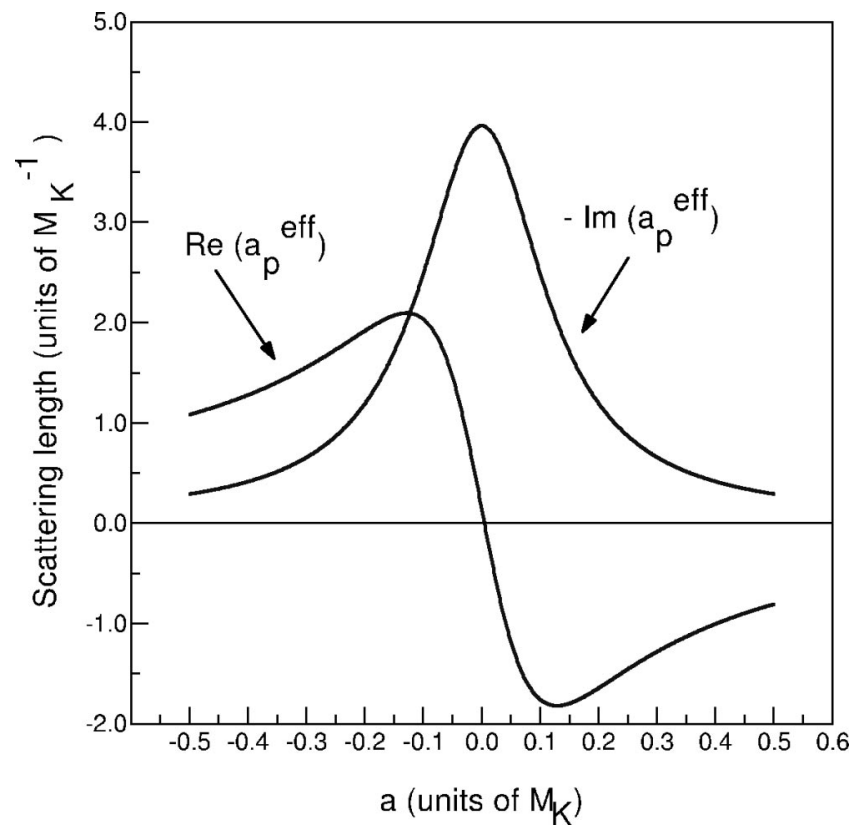

FIG. 4. Real and imaginary parts of the complex scattering length $a_{p}^{e f f}$ as a function of the parameter $a$ of the isoscalar Bargmann potential. The remaining parameters are as in Table I. The $f_{0}(980)$ ground state becomes unbound as $\operatorname{Re}\left(a_{p}^{\text {eff }}\right)$ passes through zero from positive to negative values.

the absence of Coulomb interactions, and $a_{n}^{\text {eff }}=\left(a_{0}^{\text {eff }}\right.$ $\left.-a_{1}^{e f f}\right) / 2$. One can show from Eq. (63) that the sign of $\operatorname{Re}\left(a_{I}^{\text {eff }}\right)$ is determined by the sign of the "bare" scattering length $a_{I}$ given by Eq. (25) in the absence of absorption, and hence the sign of the parameter $a$ of the Bargmann potential that controls the existence $(a<0)$ or not $(a>0)$ of a bound state in channel $I ; \operatorname{Im}\left(a_{I}^{e f f}\right)$ is independent of this sign. Figure 4 illustrates how the real and imaginary parts of $a_{p}^{\text {eff }}$ would vary should this parameter vary (i.e. the binding energy vary) in the isoscalar channel. The remaining parameters for this illustration have been kept the same as in Table I. However, the effects for small variations in the binding energy from real to virtual around zero are not large: for example simply switching the sign of $a$ to $+0.1936 M_{K}$ in Table I (thereby also unbinding the isoscalar channel), causes $\sigma_{\text {elas }}$ to decrease by $\sim 20 \%$ from 106 to $86 \mathrm{mb}$ while $\sigma_{a}$ remains unchanged.

Calculations of the total elastic, reaction, and $v$ times the annihilation cross sections based on Eqs. (57) and (58) are shown in Fig. 5. However, as there are as yet no reported measurements of $K \bar{K}$ scattering with which to compare any of these cross sections, we have attempted to use detailed balance to derive the $\pi^{+} \pi^{-} \rightarrow K \bar{K}$ cross section, for which measurements do exist [27]. In this case the latter cross section can be exactly related to the $I=0$ absorption cross section for $K \bar{K} \rightarrow \pi \pi$ defined by Eq. (59) as follows [see Eq. (A22)]:

$$
p^{2} \sigma_{a}\left(\pi^{+} \pi^{-} \rightarrow K \bar{K}\right)=\frac{2}{3} k^{2} \sigma_{a}^{(0)}=\frac{2 \pi}{3}\left(1-\eta_{0}^{2}\right)
$$

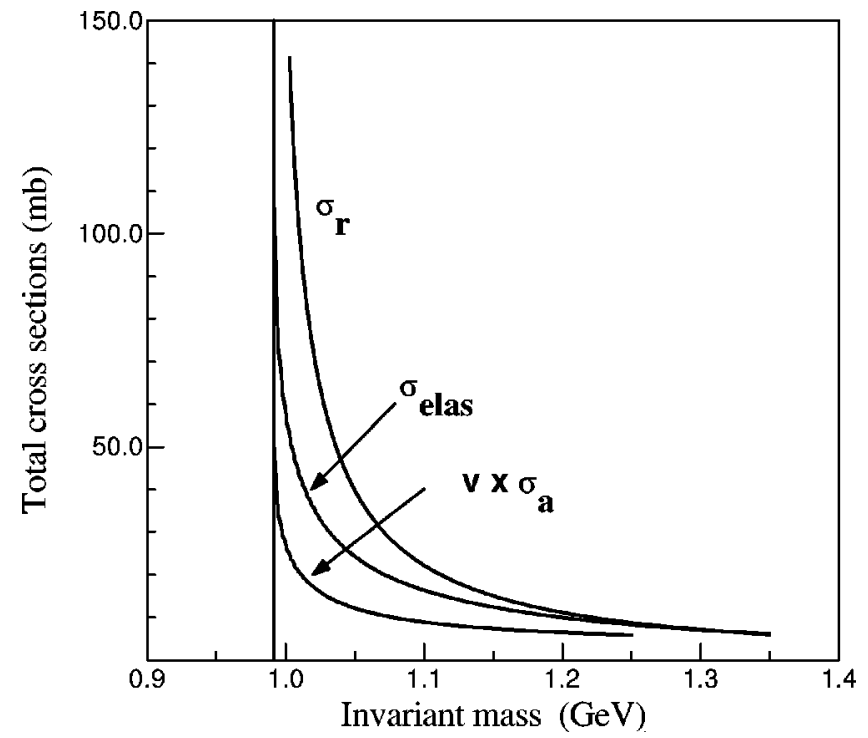

FIG. 5. Calculated total $s$-wave elastic, reaction and $v \times$ the annihilation cross sections as a function of the invariant mass $P_{0}$ of the colliding kaon-antikaon pair. The vertical line indicates the threshold value at $2 M_{K}$.

where $\eta_{0}=\left|S_{0}(k)\right|$ is the $K \bar{K}$ inelasticity, and where $p$ and $k$ are the relative momenta of the pion or kaon pair of the same total CM energy.

The two curves marked (a)+(b) in Fig. 6 (the upper one includes Coulomb distortion [55] via the factor $\left|f_{c}^{(-)}(k, d)\right|^{-2} \approx 2 \pi \eta[\exp (2 \pi \eta)-1]^{-1}$ taken from Eq. (A19) of the Appendix for $k d \sim \mu \alpha d \ll 1$ ) show the inferred results for $\sigma_{a}\left(\pi^{+} \pi^{-} \rightarrow K \bar{K}\right)$, where they are compared with $\pi^{+} \pi^{-}$ scattering data [27,28], and the fit, curve (c), to these data using a parametrized $\pi \pi$ scattering amplitude with parameters extracted from these experiments. The extraction procedure is documented in detail in [27]. A coupled channel treatment of the $\pi \pi-K \bar{K}$ system leads to similar results [20,23,30] (especially [30]) shown by curves (d) and (e).

Figure 6 clearly shows that the strong enhancement of the cross section near threshold is not adequately reproduced by the pure molecular picture. The same remark holds for the calculated $\pi \pi$ inelasticity, discussed below, that is correspondingly somewhat too large near threshold (Fig. 7).

The pure molecular picture agrees reasonably well with the result obtained by that coupled channel calculation of [30] which omits the bare scalar meson state $f_{0}^{\prime}$ [curve (d)]. This numerical finding justifies the assumptions made in order to obtain a simple analytical model. In order to obtain a good agreement with the data, the introduction of an explicit bare scalar meson state is necessary [curve (e)], however.

The parametrized $\pi \pi$ scattering amplitudes as taken from experiment [27] [curve (c)] assume the existence of a pole on the second Riemann sheet just below the $K \bar{K}$ threshold at [997 $\pm 6-(27 \pm 8) i)] \mathrm{MeV}$ in the $\pi \pi$ scattering amplitude. The experimentally inferred value of this pole lies reasonably close to the value of the pole on the second sheet for a decaying $f_{0}(980)$ bound state given in Table II, that has been calculated from the very different points of view presented here and elsewhere [6]. 


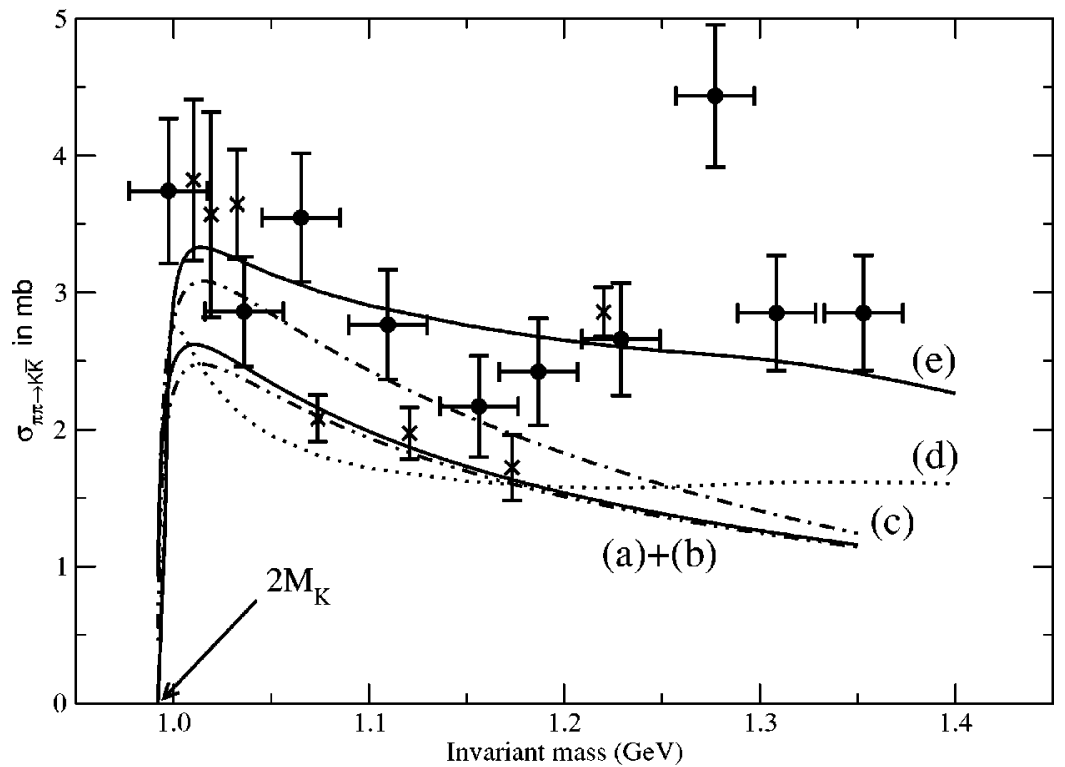

FIG. 6 . The $\pi^{+} \pi^{-} \rightarrow K \bar{K}$ cross section given by detailed balance, Eq. (66), as a function of the $K \bar{K}$ invariant mass $P_{0}$. The two curves designated (a) + (b) give the results with (upper curve) and without (lower curve) Coulomb distortion. Also shown are the $\pi^{+} \pi^{-} \rightarrow K \bar{K}$ experimental data [27] (closed circles) and [28] (crosses). Curve (c) is a fit to these data using the parametrized scattering matrix of [27]. Curves (d) and (e) correspond to coupled channel calculations of the $\pi^{+} \pi^{-} \rightarrow K \bar{K}$ cross section with [curve (e)] and without [curve (d)] $f_{0}^{\prime}$ mixing.

A further experimental check on these calculations is provided by comparing with the data [29] for the $\pi \pi$ isoscalar inelasticity parameter $\eta_{0}(\pi \pi)$. Since we have assumed that only coupling between the $\pi \pi$ and $K \bar{K}$ channels is important, this paramater equals the $K \bar{K}$ inelasticity parameter $\eta_{0}(K \bar{K})=\left|S_{0}(k)\right|$ that may be calculated directly from Eq. (60). The resulting prediction for $\eta_{0}(\pi \pi)$ is given in Fig. 7. For comparison we also show the behavior of $\eta_{0}(\pi \pi)$ that follows from a fully relativistic treatment of the pion loop integral under Pauli-Villars regularization with cutoff $\Lambda_{P V}$ $=4 \mathrm{GeV}$ (the result is rather insensitive to the actual choice of cutoff). There is no difference in the two calculations near threshold. However, the relativistic version becomes more elastic than the nonrelativistic calculation with increasing CM energy. long range Coulomb potential. In the following we construct the Jost function that determines the bound states and decay widths of kaonium, that may then be calculated without approximation. The Hamiltonian now also includes the Coulomb field $V_{c}=\alpha Q \bar{Q} / r, \quad(Q, \bar{Q}$ are particle, antiparticle charge operators) that breaks isospin. However, due to the very disparate length scales $\sim M_{K}^{-1}$ and $\sim\left(\alpha M_{K}\right)^{-1}$ over which the strong and much weaker Coulomb interactions operate, isospin breaking is essentially confined to that region of space $r \geqslant d$ outside the range of the strong potential. In this region the wave function reads

$$
\frac{1}{r}\left(\varphi_{p}\left|K^{+} K^{-}\right\rangle+\varphi_{n}\left|K^{0} \bar{K}^{0}\right\rangle\right), \quad r \geqslant d
$$

\section{KAONIUM}

Besides the isoscalar $K \bar{K}$ bound system, the $K^{+} K^{-}$comwhere $\varphi_{p}$ and $\varphi_{n}$ describe the $s$-wave radial motion of $K^{+} K^{-}$and $K^{0} \bar{K}^{0}$ separately in the external region,

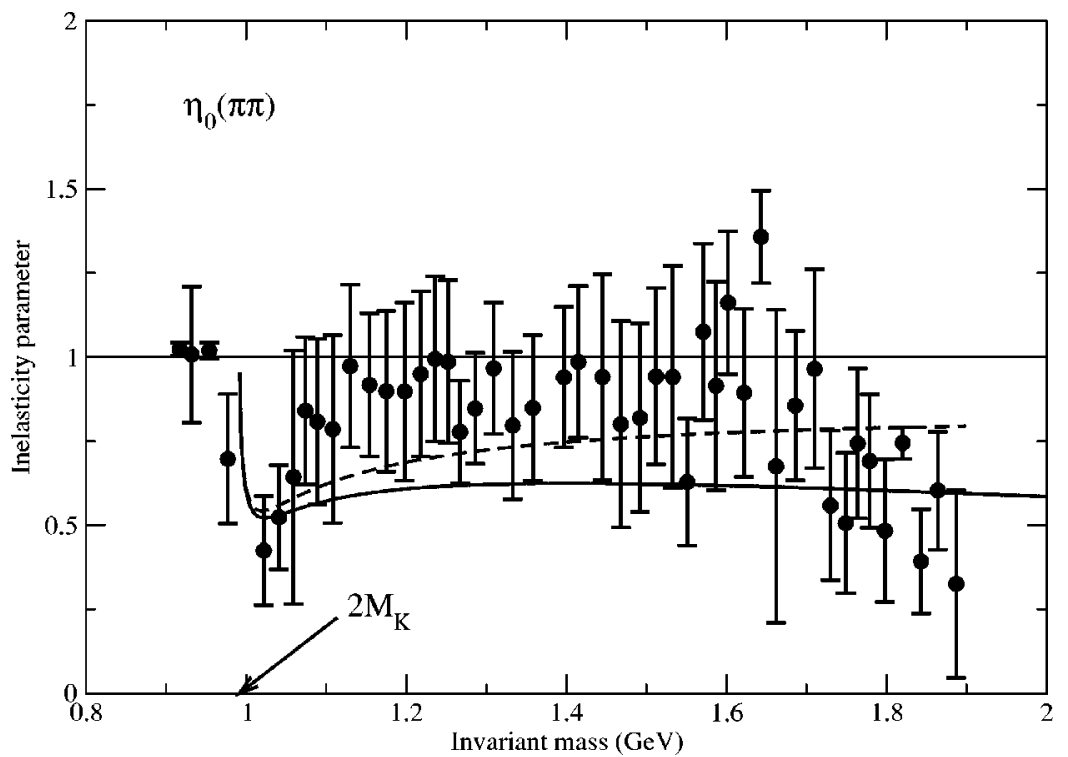

FIG. 7. The $\pi \pi$ inelasticity parameter $\eta_{0}(\pi \pi)$ as a function of the CM energy. The experimental data are taken from [29]. The upper curve shows the behavior of the inelasticity given by a fully relativistic calculation of the pion loop integral. 


$$
\begin{array}{r}
\left(\frac{d^{2}}{d r^{2}}+k^{2}-M_{K} V_{c}\right) \varphi_{p}=0, \quad r>d \\
\left(\frac{d^{2}}{d r^{2}}+k^{2}\right) \varphi_{n}=0, \quad r>d
\end{array}
$$

Inside of this range we can ignore isospin breaking entirely due to the dominance of the strong isospin conserving interaction (no internal mixing approximation $[48,49]$ ) and work with states of good isospin. To obtain the Jost function we therefore need to join the linear combinations $-\left(\varphi_{p}\right.$ $\left.\pm \varphi_{n}\right) / \sqrt{2}$ corresponding to $I=0$ and 1 in the external region $r \geqslant d$ onto the known solutions for the complex potentials in Eq. (33) in the internal region $r \leqslant d$. The latter are given by $\varphi(k, r)$ of Eq. (A5) with the appropriate values of $a, b$ and $\zeta$ taken from Table I for each isospin channel.

For $r>d$ the solution describing the scattering of a $K^{+} K^{-}$ pair is given by the standard expression

$$
\varphi_{p}(r)=f_{c}^{(-)}(k, r)-S(k) e^{2 i \sigma} f_{c}^{(+)}(k, r) .
$$

The $f_{c}^{(-)}(k, r)$ and $f_{c}^{(+)}(k, r)=f_{c}^{*(-)}(k, r)$ represent incoming (outgoing) waves in the Coulomb field, $S(k)$ is the scattering matrix of non-Coulombic origin, $\sigma=\arg \Gamma(1+i \eta)$ the Coulomb phase shift and $i \eta=\mu \alpha / i k$ the Coulomb parameter for attractive interactions; $\mu=M_{K} / 2$ is the reduced mass. The $f_{c}^{( \pm)}(k, r)$ are proportional to Whittaker functions. One has [50]

$$
\begin{aligned}
f_{c}^{(-)}(k, r)= & e^{\pi \eta / 2} W_{i \eta, 1 / 2}(2 i k r) \\
\approx & \frac{e^{\pi \eta / 2}}{\Gamma(1-i \eta)}\{1-i k r-2 \mu \alpha r \\
& \times[\ln 2 i k r+\psi(1-i \eta)+2 \gamma-1]+\cdots\}, \\
& k r \rightarrow 0
\end{aligned}
$$

where the small $k r$ behavior of $f_{c}^{(-)}(k, r)$ involves [51] the standard $\psi$ function, and $\gamma=0.57721$ is the Euler constant.

The $S$ matrix in Eq. (69) is determined by the logarithmic derivative $\zeta_{p}=\varphi_{p}^{\prime} / \varphi_{p}$ of $\varphi_{p}$ at $r=d$. In detail,

$$
S(k)=e^{-2 i(\sigma-\rho)}\left(\frac{\zeta_{p}-\zeta_{c}}{\zeta_{p}-\zeta_{c}^{*}}\right)
$$

where $\zeta_{c}=f_{c}^{\prime(-)} / f_{c}^{(-)}$is the logarithmic derivative of the incoming Coulomb wave at $r=d$, and $\rho=\arg f_{c}^{(-)}(k, d)$. Thus the Jost function describing kaonium is proportional to $\zeta_{p}$ $-\zeta_{c}$. To find it [49] we determine the value of $\zeta_{p}$ by matching $\varphi_{p} \pm \varphi_{n}$ onto the internal solutions $\varphi_{0}$ and $\varphi_{1}$ of good isospin, where $\varphi_{n} \sim \exp (i k r)$ is a pure outgoing wave [52]. Due the large difference in space behavior of the strong $(b r \gg 1)$ and Coulombic $(k r \ll 1)$ wave functions near $r \sim d$ for momenta $k \sim \mu \alpha$ of interest for kaonium, the $\varphi(k, r)$ have already reached their asymptotic behavior, while the $f_{c}^{( \pm)}(k, r)$ are still given by their small $k r$ approximation.
The matching of value and derivative at $r=d$ is therefore particularly simple to perform. One finds

$$
\frac{1}{\zeta_{p}}=\frac{1}{2}\left(\frac{1}{\zeta_{0}}+\frac{1}{\zeta_{1}}\right)
$$

where the logarithmic derivatives $\zeta_{I}=\varphi_{I}^{\prime} / \varphi_{I}$ of the internal wave functions carrying good isospin $I$ are

$$
\zeta_{I}=i k\left(\frac{f(k) e^{i k d}+f(-k) e^{-i k d}}{f(k) e^{i k d}-f(-k) e^{-i k d}}\right) .
$$

The eigenvalue condition $\zeta_{p}=\zeta_{c}$ that determines complex energies of kaonium then reads

$$
1-\frac{1}{2} \zeta_{c}\left(\frac{1}{\zeta_{0}}+\frac{1}{\zeta_{1}}\right)=0
$$

This equation contains no free parameters. The Jost functions $f(k)$ in the $\zeta_{I}$ all have predetermined constants for each isospin channel as given in Table I, and come from Eq. (35) if annihilation is included, or Eq. (22) if it is not.

It is useful to express the roots of Eq. (74) in the form $k_{\lambda}=-i \lambda \mu \alpha$ since then $\lambda^{-1} \rightarrow 1,2,3, \ldots$ for a pure Coulomb field, and also to recognize that the logarithmic derivatives $\zeta_{I}$ can be replaced by their zero momentum values with impunity because $k$ is always $O(\mu \alpha)$ for kaonium. Then

$$
\frac{1}{\zeta_{I}} \approx-\left(a_{I}^{e f f}-d\right)+O\left(1 / \zeta^{2}\right)
$$

in terms of the complex scattering length of Eq. (63).

Using these forms for the $\zeta_{I}$, the eigenvalue condition (74) can be reformulated in terms of the complex scattering length $a_{p}^{e f f}$ for $K^{+} K^{-}$scattering as

$$
a_{p}^{e f f}=\frac{1}{2}\left(a_{0}^{e f f}+a_{1}^{e f f}\right)=\left(1-\frac{1}{\zeta_{c} d}\right) d .
$$

This leads to an eigenvalue equation of the KudryavtsevPopov (KP) type [31],

$$
1 / a_{p}^{e f f}=2 \mu \alpha\left[\psi(1-1 / \lambda)+\frac{1}{2} \lambda+\ln (2 \lambda \mu \alpha d)+2 \gamma\right] .
$$

The logarithmic derivative $\zeta_{c}(k)$ of the Coulomb wave function has been calculated at $k_{\lambda}=-i \lambda \mu \alpha$ from Eq. (70).

The resulting complex energy spectrum for kaonium is then given by

$$
E_{\lambda}-\frac{i}{2} \Gamma_{\lambda}=-\frac{1}{4} \lambda^{2} M_{K} \alpha^{2}
$$

The solutions of the KP equation are dominated by the $\psi$ function on the right hand side that has poles at $\lambda=1 / n$, for integer $n$. These poles just reproduce the pure Coulomb spectrum of kaonium. The strong interaction changes this behavior by moving $\lambda$ off these poles to $\lambda=(n+\delta)^{-1}$, where $\delta$ is 
TABLE IV. Complex level shifts and lifetimes due to the strong interactions away from the point Coulomb value $-\frac{1}{4} \alpha^{2} M_{K} / n^{2}=-6.607 / n^{2} \mathrm{keV}$ for the ground and excited $s$-states of kaonium. Numerical solutions of the Kudryavtsev-Popov equation have been generated for the $K^{+} K^{-}$scattering length $a_{p}^{\text {eff }}$ $=(1.940-1.199 i) M_{K}^{-1}$ given by Eq. (79). The matching radius is $d \sim 3 M_{K}^{-1}$. The real part of $\lambda$ lies close to the sequence $1 / 1,1 / 2,1 / 3,1 / 4, \ldots$ for a pure Coulomb spectrum.

\begin{tabular}{lccc}
\hline \hline Level & $\lambda$ & $\Delta E-i \Gamma / 2(\mathrm{keV})$ & Lifetime $\left(\times 10^{-18} \mathrm{sec}\right)$ \\
\hline 3rd & $0.2491+0.0005 i$ & $0.003-0.002 i$ & 199 \\
2nd & $0.3318+0.0009 i$ & $0.007-0.004 i$ & 84 \\
1st & $0.4965+0.0020 i$ & $0.023-0.013 i$ & 25 \\
Ground & $0.9863+0.0079 i$ & $0.180-0.103 i$ & 3.2 \\
\hline \hline
\end{tabular}

complex and not necessarily small. In Table IV we list a series of exact numerical eigenvalues $\lambda$ of the KP equation, and the associated complex energy spectrum for kaonium. These computations use the value

$$
a_{p}^{e f f}=(1.940-1.199 i) M_{K}^{-1}=(0.77-0.48 i) \mathrm{fm}
$$

for the complex scattering length calculated from Eq. (63) plus the data given in Table III. Notice that the $a_{0}(980)$ admixture introduced via the isovector scattering length $a_{1}$ only contributes to the real part of the $K^{+} K^{-}$effective scattering length that enters into the calculation of the level shifts and decay widths of kaonium. The imaginary part is entirely determined by the isoscalar piece of the strong interactions. However, due to the nonlinear nature of the eigenvalue problem, the isovector contribution nevertheless influences both the real and imaginary parts of the resulting energy spectrum. Only in the perturbative limit (to be described below) does the $a_{0}(980)$ mode contribute solely to the level shifts.

For comparison we have also used the isospin zero and one scattering lengths $\hat{a}_{0}^{\text {eff }}=(0.86-1.82 i)$ fm and $\hat{a}_{1}^{\text {eff }}$ $=(0.06-0.77 i) \mathrm{fm}$, based on a recent investigation [30] into the effects of a high lying scalar isoscalar quark antiquark configuration (which we refer to as $f_{0}^{\prime}$ ) mixed into the $K \bar{K}$ molecular ground state. Here the effect of the $\pi \eta$-channel is included too. The $K^{+} K^{-}$scattering length then becomes

$$
\hat{a}_{p}^{e f f}=(1.156-3.268 i) M_{K}^{-1}=(0.46-1.30 i) \mathrm{fm}
$$

instead of Eq. (79).

The predictions for kaonium are given in Tables IV and $\mathrm{V}$, while Table VI summarizes the decrease in lifetime of the ground state as the complexity of the $K^{+} K^{-}$interaction is increased. In particular, Table VI shows that the lifetime of kaonium is reduced further by about a factor of three once annihilation in the isovector channel is introduced via the $\pi \eta$ decay of the $a_{0}(980)$. These results are qualitatively similar to those given by other approaches to the problem of kaonium formation and decay [53]. One notes from Tables IV and $\mathrm{V}$ that $\operatorname{Re} \lambda<1 / n$, corresponding to a positive shift of the Coulomb level when the strong interaction supports a bound state (the sign of the real part of the scattering length determines the sign of the level shift). In addition to causing repulsive shifts of the Coulomb levels the strong field introduces a decay width due to $K^{+} K^{-} \rightarrow 2 \pi$ annihilation with a lifetime of $\sim 10^{-18} \mathrm{sec}$ [the two-photon annihilation lifetime at $O\left(M_{K}^{-1} \alpha^{-5}\right) \sim 10^{-13} \mathrm{sec}$, or one in $10^{5}$ decays, is too long to compete]. It also introduces an extra node into all the eigenfunctions of kaonium. This means that the kaonium levels represent excited states in the combined Coulomb plus strong fields of the $K \bar{K}$ system. As will be discussed in more detail below, the occurrence of this node is responsible for making the kaonium lifetime two orders of magnitude shorter than the $\sim 10^{-16} \mathrm{sec}$ it would have been had the $K^{+} K^{-}$pair only been bound by a Coulomb field [54].

Let us briefly indicate how these features come about. The appearance of the additional radial node is required by the fact that the $1 s$ state of relative motion has been usurped by the $K \bar{K}$ ground state in the strong potential. So the ground and excited levels of kaonium have to have a $2 s, 3 s \ldots$ character with one additional node. This node is always positioned in the close proximity of the $K^{+} K^{-}$scattering length, independent of the excitation energy. It is real or virtual depending on whether the strong interaction supports a real $\left(a_{p}>0\right)$ or a virtual $\left(a_{p}<0\right)$ bound state. This comes about

TABLE V. A repeat of the calculations described in Table IV, but for the scattering length $\hat{a}_{p}^{e f f}$ $=(1.156-3.268 i) M_{K}^{-1}$ of Eq. (80) which has been taken from the model of Ref. [30] that includes $f_{0}^{\prime}$ mixing and the $+\pi \eta$-channel. This choice leads to lifetimes of about a factor three shorter than those given in Table IV.

\begin{tabular}{lccc}
\hline \hline Level & $\lambda$ & $\Delta E-i \Gamma / 2(\mathrm{keV})$ & Lifetime $\left(\times 10^{-18} \mathrm{sec}\right)$ \\
\hline 3rd & $0.2494+0.0014 i$ & $0.002-0.005 i$ & 71 \\
2nd & $0.3322+0.0025 i$ & $0.005-0.011 i$ & 30 \\
1st & $0.4975+0.0056 i$ & $0.017-0.037 i$ & 8.9 \\
Ground & $0.9899+0.0223 i$ & $0.137-0.291 i$ & 1.1 \\
\hline \hline
\end{tabular}


TABLE VI. A comparison of predicted lifetimes of the kaonium ground state for increasing complexity of the $K^{+} K^{-}$interaction.

\begin{tabular}{lcc}
\hline \hline & $\begin{array}{c}\text { Scattering } \\
\text { length (fm) }\end{array}$ & $\begin{array}{c}\tau\left(K^{+} K^{-} \rightarrow \pi \pi\right) \\
(\mathrm{sec})\end{array}$ \\
\hline $\begin{array}{l}\text { Free annihilation at rest } \\
+ \text { One boson exchange }\end{array}$ & $0.00-0.01 i$ & $1.2 \times 10^{-16}$ \\
$+f_{0}^{\prime}$ mixing & $0.77-0.48 i$ & $3.2 \times 10^{-18}$ \\
$+\pi \eta$-channel [30] & $0.46-1.30 i$ & $1.1 \times 10^{-18}$ \\
\hline \hline
\end{tabular}

as follows. The kaonium levels are all weakly bound at $k$ $\sim \mu \alpha$ so that their radial wave functions in the inner region all become nearly identical with the zero energy wave functions of $K \bar{K}$ in the strong potential alone. These functions [see Eq. (A15)] all have a node at $r=a_{p}$ in the effective range approximation. The remaining nodes are supplied by the Whittaker function $W_{1 / \lambda, 1 / 2}(2 \lambda \mu \alpha r)$ in the external region, $r \geqslant d$. They are almost identical with those of a pure Coulomb field bound state wave function where $\lambda=1 / n$.

The eigenfunction for the lowest state of kaonium is illustrated in Fig. 8 (with absorption suppressed). It is clear from this figure that the actual choice of the cutoff $d$ is immaterial if taken anywhere in the region where the wave function behaves linearly. A closed form for the bound state eigenfunctions $\varphi_{1 / \lambda}$ of kaonium labeled by the quantum number $\lambda^{-1}$ is given in Eq. (A13) in the Appendix. From that one calculates that the gradient at the origin is enhanced by the

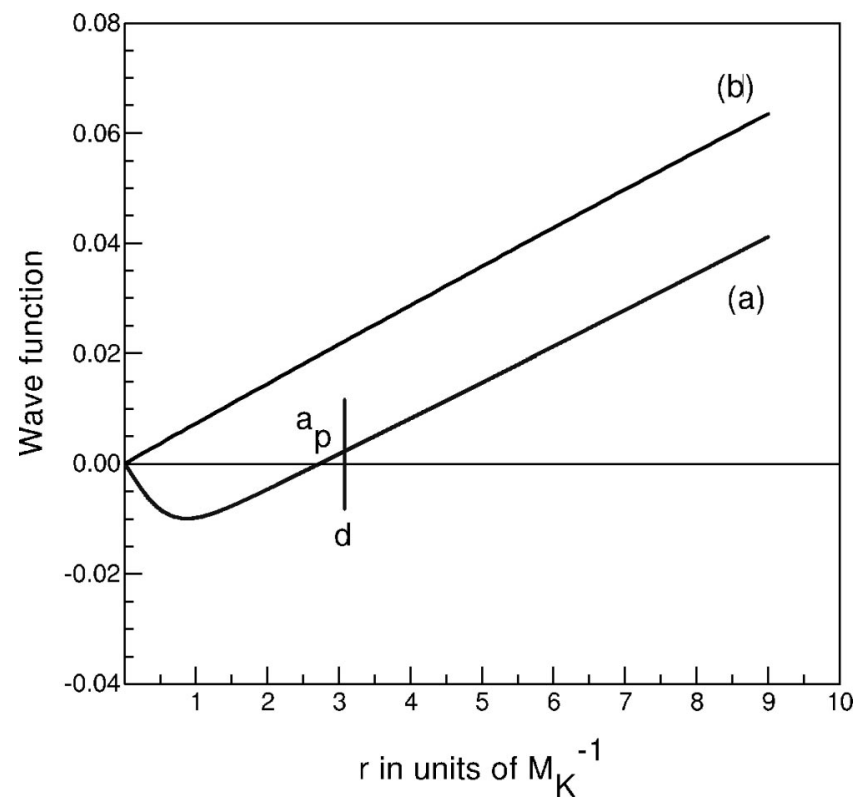

FIG. 8. Kaonium ground state radial wave functions: (a) with and (b) without the meson exchange fields present. In (a) the strong field solution, Eq. (A13), is joined smoothly at $r=d$ onto the Whittaker function $W_{1 / \lambda, 1 / 2}(2 \lambda \mu \alpha r)$ for $\lambda=0.981$. The node in this solution falls at the $K^{+} K^{-}$scattering length $a_{p}$. In (b) the solution $W_{1,1 / 2}(2 \mu \alpha r)=2 \mu \alpha r \exp (-\mu \alpha r)$ for a pure Coulomb field, $\lambda$ $=1$, is plotted for the same range of $r$. To set the scale, note that the Bohr radius of kaonium would fall at $\sim 300$ units to the right of this figure. factor $b / a$ in both isospin channels over that of the pure Coulomb state. Physically this comes about because the strong potentials draw the $K^{+} K^{-}$pair much closer together, thereby increasing the probability density of finding them at the origin by $\left(b^{2} / a^{2}\right)_{I}$ [notice $b>|a|$ from Eq. (26)] in each isospin component, where they annihilate. In the isoscalar channel, which is strongly attractive, the effect is especially marked, $\left(b^{2} / a^{2}\right)_{0} \sim 60$. On the other hand the isovector channel only supports a virtual state. Hence the concentration of probability density at the origin in this channel is much weaker, $\left(b^{2} / a^{2}\right)_{1} \sim 3$.

The effect of this factor in the isoscalar channel is quite dramatic. It increases the decay width of kaonium by two orders of magnitude (with a corresponding decrease in lifetime) over what it would have been in the absence of the strong potentials $V_{I}$. One can illustrate this qualitatively by appealing to the perturbative solution [31,37] of the KP equation for the complex energy shift. For the ground state this reads

$$
\begin{aligned}
\Delta E-\frac{i}{2} \Gamma & \approx \frac{1}{2} M_{K}^{2} \alpha^{3} a_{p}^{e f f} \\
& =\frac{1}{2} M_{K}^{2} \alpha^{3} a_{p}-\frac{1}{2} M_{K}^{2} \alpha^{3} \xi^{-1}\left[\frac{1}{2}\left(\frac{b^{2}}{a^{2}}\right)_{0}\right]
\end{aligned}
$$

that equals either $(0.187-0.116 i) \mathrm{keV}$, or $(0.187$ $-0.164 i) \mathrm{keV}$ depending on whether the value (79) or its low absorption version $\xi \gg 1$ taken from Eq. (63),

$$
a_{p}^{e f f} \approx \frac{\left(a_{0}+a_{1}\right)}{2}-\frac{i}{2}\left(\frac{b^{2}}{a^{2} \xi}\right)_{0}=(1.940-1.703) M_{K}^{-1}
$$

is used for the effective scattering length. The $a_{I}$ are the bare scattering lengths of Eq. (25) as before. The last approximation places the role of the isoscalar probability density admixture $\left(b^{2} / a^{2}\right)_{0}$ in the kaonium ground state explicity in evidence.

Neither approximation is very accurate in comparison with the exact solution of $(0.181-0.107 i) \mathrm{keV}$, but can be used to illustrate the effect of strong interactions decreasing the lifetime of kaonium against what it would have been in a pure Coulomb field. One sees this as follows. The decay width in Eq. (81) is directly related to the "annihilation at rest" cross section

$$
\begin{aligned}
\sigma_{a} & \approx-\frac{4 \pi}{k} \operatorname{Im}\left(a_{p}^{e f f}\right)=\frac{4 \pi}{k} \frac{1}{2}\left[\frac{b^{2} \xi}{a^{2} \xi^{2}+\left(b^{2}-a^{2}\right)^{2}}\right]_{0} \\
& \approx \frac{4 \pi}{k \xi}\left[\frac{1}{2}\left(\frac{b^{2}}{a^{2}}\right)_{0}\right]
\end{aligned}
$$

as $\Gamma=v \phi^{2}(0) \sigma_{a}$, upon multiplication by the flux factor $v$ $\times \phi^{2}(0)$ where $\phi^{2}(0)=M_{K}^{3} \alpha^{3} / 8 \pi$ gives the probability per unit volume for finding the $K^{+} K^{-}$pair at the origin when 
bound by the Coulomb field alone; $v=k / \mu$ is their relative velocity. On the other hand when the strong potential is absent, the scattering lengths $a_{0}^{e f f} \rightarrow-i / \xi$ and $a_{1}^{\text {eff }} \rightarrow 0$ are pure imaginary and zero respectively, so that $a_{p}^{e f f} \rightarrow-i / 2 \xi$ $=-0.028 i M_{K}^{-1}$, and the corresponding free annihilation cross section becomes

$$
\sigma_{a, \text { free }}=\frac{4 \pi}{k \xi}\left(\frac{1}{2}\right) \approx \sigma_{a} / \theta, \quad \theta \sim 42 \text { to } 60
$$

if we use the unexpanded or expanded version of $\sigma_{a}$ in Eq. (83). The factor $\theta$ reflects the modification of the kaonium ground state due to the strong interactions.

In this case there is no energy shift at all, and the decay half-width is $\frac{1}{2} \Gamma_{\text {free }}=0.0028 \mathrm{keV}$, leading to a lifetime of $\tau_{\text {free }}=1.2 \times 10^{-16} \mathrm{sec}$. So the $K^{+} K^{-} \rightarrow 2 \pi$ strong potential assisted annihilation lifetime drops by roughly the factor $\theta$ from $1.2 \times 10^{-16} \mathrm{sec}$ that it would have been in a pure Coulomb field, to $3.2 \times 10^{-18} \mathrm{sec}$ as given by the exact solution of the KP equation. Table VI summarizes the change in lifetime of the kaonium ground state as the complexity of the $K^{+} K^{-}$interaction changes from assuming free annihilation at rest in a Coulomb field, to including one-boson exchange, to adding $f_{0}^{\prime}$ mixing, for determining the scattering length.

We note in passing from the center expression for $\sigma_{a}$ in Eq. (83) that if the bound state occurs at zero energy ( $a$ $=0)$, the corresponding enhancement of the annihilation width is maximal. Also, while generally true of the exact solutions of the KP equation, the perturbative solution given in Eq. (81) makes it evident that the sign of the level shift goes along with the sign of the scattering length, which is positive for bound and negative for unbound or virtual states.

We close this section with a comment on to what extent the results for kaonium might be considered as "parameterfree." Apart from specifying a Lagrangian with the attendant assumption of $S U(3)$ symmetry that fixes the various coupling constants in terms of $g_{\rho \pi \pi}$, that we in turn fixed from the KSRF relation, the only arbitrary parameter in these calculations was the form factor cutoff $\Lambda$ introduced in Sec. III A. The choice $\Lambda=4 \mathrm{GeV}$, that was also used in Ref. [20], led to calculated scattering lengths and effective ranges for the $K \bar{K}$ system, that were then duplicated by constructing an equivalent Bargmann potential that depended on two parameters $a$ and $b$. This in turn gave predictions for the mass and width of a bound $K \bar{K}$ molecular state in reasonable accord with the known experimental mass and width of the $f_{0}(980)$ scalar meson. However, as already demonstrated in Table II, one can turn the argument around without materially affecting any of our conclusions for kaonium, by asserting that the Bargmann parameters $a$ and $b$ are actually predetermined from experiment in the strong interaction sector, thus leaving the kaonium sector without any free parameters at all.

Similar remarks hold true for the calculated cross sections shown in Figs. 5 and 6. Once $a$ and $b$ have been fixed from experiment or otherwise, no further adjustable parameters enter into these calculations. The actual values of $a$ and $b$ as determined by any of the five alternatives shown in Table II do not in fact differ very much.

\section{DISCUSSION}

In this investigation we have shown that one-meson exchange potentials derived from a standard $S U(3)_{V}$ $\times S U(3)_{A}$ Lagrangian in the nonrelativistic limit are sufficient to bind $K \bar{K}$ into a kaonic molecule with a mass and decay width that closely match the experimental values of the $f_{0}(980)$ isoscalar meson. We have also shown that the same potentials, in combination with detailed balance, lead to a prediction of the $\pi^{+} \pi^{-} \rightarrow K \bar{K}$ cross section that is too low especially near threshold.

We have further demonstrated that there are specific, experimentally observable effects in the energy level shifts and decay properties of the kaonium spectrum, in the presence of a $K \bar{K}$ molecular ground state. The existence of such a bound $K \bar{K}$ pair would be reflected in positive energy level shifts in the kaonium Coulomb spectrum (due to level repulsion), plus enhanced $K^{+} K^{-} \rightarrow 2 \pi$ decay widths. On the other hand, unbound or virtually bound (and therefore weakly correlated) $K \bar{K}$ pairs produce negative energy level shifts and a weak enhancement of the decay widths. Since the sign of the level shifts and the enhancement of the decay widths go together with the presence or absence of a strongly correlated $K \bar{K}$ pair in the ground state, measurements of the level shifts and decay widths in kaonium could provide another probe for investigating to what extent the $f_{0}(980)$ meson ground state has a $K \bar{K}$ molecular component. From the experimental point of view it is important to stress that, as for protonium [31] which has a very similar decay width, the $1 s-2 s$ level spacing of $\sim 5.1 \mathrm{keV}$ in kaonium is many times the estimated decay widths $\leqslant 200 \mathrm{eV}$, so that these levels remain distinguishable in the presence of the $2 \pi$ decay channel.

Notwithstanding the results reported in [45], we again stress the conflict that exists between the pure molecular description of the $f_{0}(980)$ ground state and the calculated $2 \gamma$ decay width. In [45] a binding energy of $10 \mathrm{MeV}$ for the kaonium molecule was assumed, that leads to a width of $\sim 0.6 \mathrm{keV}$. However, most recent data show a resonance energy that corresponds to a pole in the complex plane, which translates into a binding energy of 18 to $20 \mathrm{MeV}$ for the molecular potential. This increase in binding energy enhances the value of the spatial wave function at the origin by a factor of three, see Fig. 9. One way of understanding the origin of this discrepancy is to generalize the molecular picture of the $f_{0}(980)$ to a superposition of a $K \bar{K}$ component and a hard component. In the Jülich model [20] a $f_{0}^{\prime}$ state has been considered in addition to the pure molecule in order to reproduce the large inelasticities of the $\pi \pi$ phase shifts in the vicinity of the $K \bar{K}$ threshold. The $f_{0}^{\prime}$ state may be interpreted as either a $q \bar{q}$ or as a $q^{2} \bar{q}^{2}$ configuration. As pointed out by Achasov [5] and confirmed by current algebra [43], the large decay width of the former into two pions may disqualify a $q \bar{q}$ interpretation of the additional component $f_{0}^{\prime}$. 


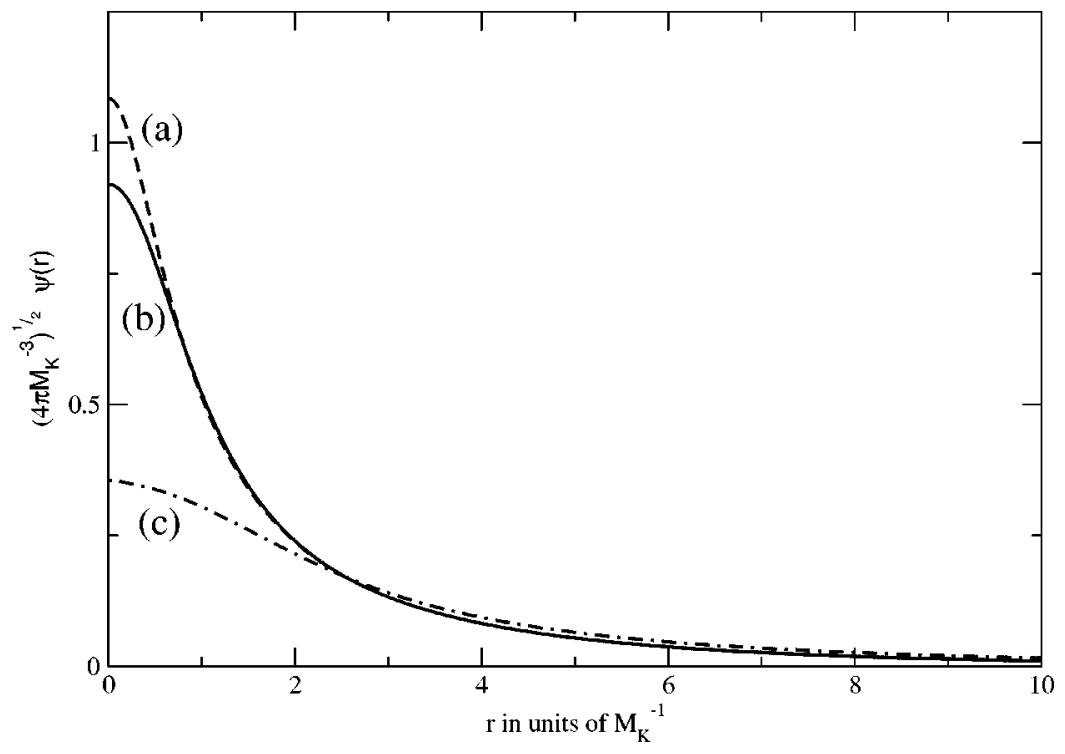

FIG. 9. Comparison of the bound state wave function obtained numerically [curve (a)] for the isoscalar one-boson exchange potential of Eq. (17), with that given by the analytical solution, Eq. (A10), for the equivalent Bargmann potential [curve (b)]. Curve (c) shows the wave function in a Gaussian potential adjusted to give a binding energy of $10 \mathrm{MeV}$ [45].
The effect of the $f_{0}^{\prime}$ component in the $f_{0}(980)$ meson introduced in the Jülich model is to reduce the lifetime of kaonium from $3.2 \times 10^{-18} \mathrm{sec}$ to $1.1 \times 10^{-18} \mathrm{sec}$. The presently available data concerning the inelasticities of the $\pi \pi$ phase shifts, and in particular the $\pi^{+} \pi^{-} \rightarrow K \bar{K}$ cross sections, do not allow one to disentangle the components of the $f_{0}(980)$ structure in a model independent way. High resolution data for two kaon production near to the threshold will be undertaken at COSY in the near future.

\section{ACKNOWLEDGMENTS}

One of us (R.H.L.) would like to thank Professor Speth of the Institut für Kernphysik Theory Division, Forschungszentrum, Jülich, for the exceptional hospitality of the institute, and for financial support. It is a pleasure to thank M. Büscher, D. Gotta, C. Hanhart and A. E. Kudryavtsev for useful discussions.

\section{APPENDIX A}

\section{Isospin phase conventions}

In order to construct two-meson states of good isospin using conventional Clebsch-Gordan coefficients the following phase conventions are useful.

If one chooses [38] as basis states $\left|\pi^{ \pm}\right\rangle=\mp|\pi, \pm 1\rangle$, and $\left|\pi^{0}\right\rangle=|\pi, 0\rangle$ for charged and neutral pions, the two-pion state of good isospin $\left|I=0, I_{3}=0\right\rangle$ is, for example,

$$
\begin{aligned}
\left|(\pi \pi)_{0}\right\rangle= & -\sqrt{\frac{1}{3}}\left[\left|\pi^{+}(1) \pi^{-}(2)\right\rangle\right. \\
& +\left|\pi^{-}(1) \pi^{+}(2)\right\rangle \\
& \left.+\left|\pi^{0}(1) \pi^{0}(2)\right\rangle\right] .
\end{aligned}
$$

Since the pions are identical bosons in the isospin basis, one has to include a space part of the two-pion wave function that is symmetric in particle label interchange to accompany

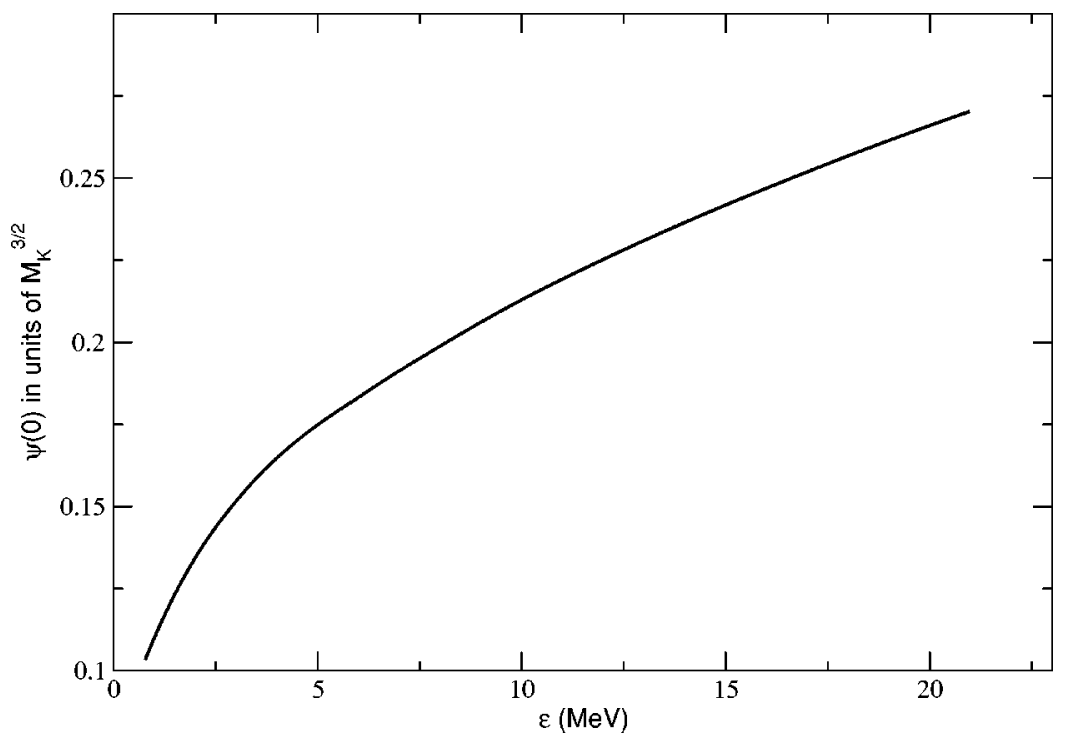

FIG. 10. Variation of the spatial wave function $\psi(0)$, Eq. (A10), with binding energy. 
$\left|I=0, I_{3}=0\right\rangle$. The normalization of the symmetrized product of the two-pion space wave function introduces an additional factor $n_{s}=1 / \sqrt{2}$.

For the kaon and antikaon isospin doublet and antidoublet the analogous convention is, in matrix form,

$$
K=\left(\begin{array}{c}
K^{+} \\
K^{0}
\end{array}\right), \quad \bar{K}=\left(\begin{array}{c}
\bar{K}^{0} \\
-K^{-}
\end{array}\right) \text {. }
$$

Consequently the isoscalar and isovector combinations $\mid I$ $\left.=0, I_{3}=0\right\rangle$ and $\left|I=1, I_{3}=0\right\rangle$ are

$$
\begin{aligned}
& \left|(K \bar{K})_{0}\right\rangle=-\frac{1}{\sqrt{2}}\left[\left|K^{+}(1) K^{-}(2)\right\rangle+\left|K^{0}(1) \bar{K}^{0}(2)\right\rangle\right] \\
& \left|(K \bar{K})_{1}\right\rangle=-\frac{1}{\sqrt{2}}\left[\left|K^{+}(1) K^{-}(2)\right\rangle-\left|K^{0}(1) \bar{K}^{0}(2)\right\rangle\right] .
\end{aligned}
$$

These relations lead immediately to the values of the isospin coefficients $C_{I}$ and $n_{s}$ quoted below Eq. (13) in the text.

\section{Wave functions for the Bargmann potentials}

We briefly summarize some results of Bargmann for constructing phase equivalent potentials. As shown in [24] the assumed form Eq. (22) for the Jost function gives rise to a family of potentials that lead to this form for $f(k)$ : the simplest one of this family is given by Eq. (23). Let $\psi(r)$ $=r^{-1} \varphi(r)$, and replace the OBE potentials by this form for $V_{I}$. Then

$$
\varphi^{\prime \prime}+\left(k^{2}-M_{K} V_{I}\right) \varphi=0
$$

for $K \bar{K} s$-wave scattering. The two linearly independent solutions $f( \pm k, r)$ for this equation that are irregular at the origin are known in closed form by construction [24,34]

$$
f(k, r)=e^{-i k r} \frac{k-i b[b \sinh (b r)+a \cosh (b r)][b \cosh (b r)+a \sinh (b r)]^{-1}}{k-i b} \rightarrow e^{-i k r}, \quad r \rightarrow \infty
$$

A general solution of Eq. (A3) can be written as a linear combination of the $f( \pm k, r)$,

$$
\varphi(k, r)=\frac{i}{2 k}[f(-k) f(k, r)-f(k) f(-k, r)]
$$

where the coefficient $f(k)$ is the Jost function. It can be shown quite generally [34] that $f(k)$ is determined by the Wronskian of $f(k, r)$ and $\varphi(k, r)$ :

$$
f(k)=W[f(k, r), \varphi(k, r)]=\left[f \varphi^{\prime}-f^{\prime} \varphi\right] .
$$

Since $W$ is independent of $r$ this can be evaluated at any convenient point, in particular at the origin. The value of $f(k)$ is determined by how $\varphi$ behaves at the origin. If $\varphi$ $\sim r$, then $f(k)=f(k, 0)=(k-i a) /(k-i b)$ as in Eq. (22). This is the usual case. On the other hand if $\varphi$ does not vanish at the origin, but instead possesses a finite logarithmic derivative there, then $f(k)$ is given by Eq. (35).

In the first case one obtains the usual scattering solution of Eq. (A3) that vanishes at the origin as

$$
\begin{aligned}
\varphi(k, r)= & \frac{\sin k r}{k}+\frac{b^{2}-a^{2}}{b^{2}+k^{2}} \frac{1}{k} \\
& \times \frac{k \sinh (b r) \cos (k r)-b \cosh (b r) \sin (k r)}{a \sinh (b r)+b \cosh (b r)} .
\end{aligned}
$$

If $a=-\kappa$ is negative, then $f(k)$ has a zero in the lower half of the complex $k$-plane, corresponding to a bound state with binding energy $\varepsilon=\kappa^{2} / M_{K}$. The associated bound state wave function is found by setting $a=-\kappa$ and $k=-i \kappa$ in Eq. (A7) to find

$$
\varphi(-i \kappa, r)=\frac{\sinh (b r)}{b \cosh (b r)-\kappa \sinh (b r)} e^{-\kappa r} .
$$

The normalization of this bound state is also available from a knowledge of the Jost function:

$$
\begin{aligned}
\int_{0}^{\infty} d r \varphi^{2}(-i \kappa, r) & =\frac{i}{4 \kappa^{2}} f(i \kappa) \frac{\partial f(k)}{\partial k} k \rightarrow-i \kappa \\
& =\frac{i}{4 \kappa^{2}} f(i \kappa) \dot{f}(-i \kappa)=\frac{1}{2 \kappa\left(b^{2}-\kappa^{2}\right)}
\end{aligned}
$$

since $f(k)=(k+i \kappa) /(k-i b)$. The normalized bound state is then given by

$$
\begin{aligned}
\psi(r)= & (\sqrt{4 \pi} r)^{-1} \sqrt{2 \kappa\left(b^{2}-\kappa^{2}\right)} \\
& \times \frac{\sinh (b r)}{b \cosh (b r)-\kappa \sinh (b r)} e^{-\kappa r} .
\end{aligned}
$$

In Fig. 9 we compare the radial eigenfunction as obtained by numerically solving for the $K \bar{K}$ isoscalar bound state in the one-boson exchange potential of Eq. (17), with the ana- 


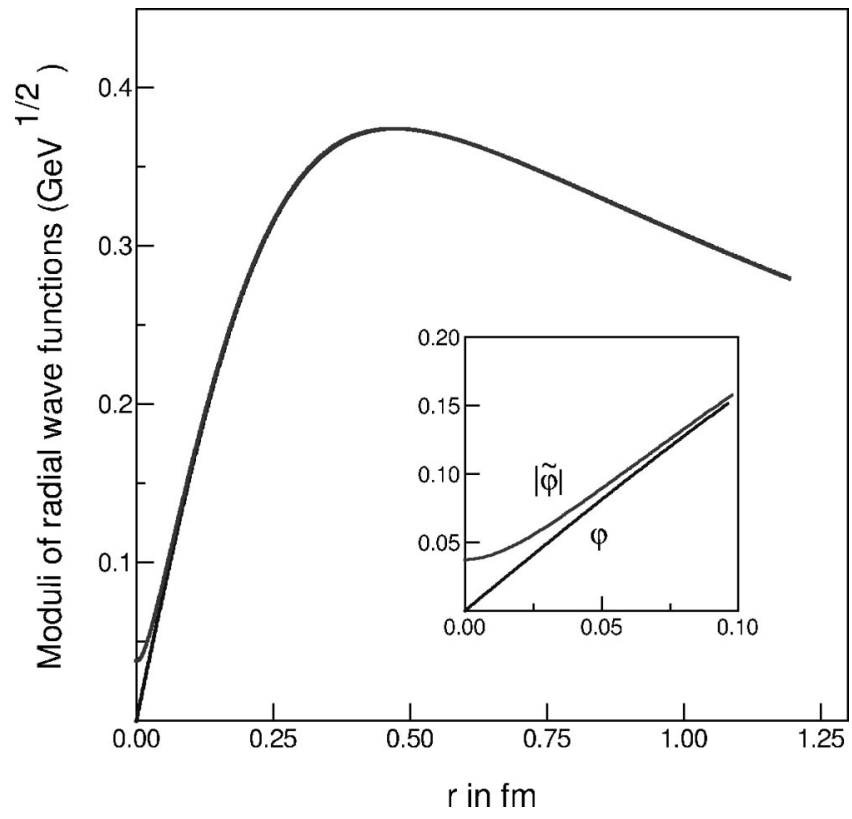

FIG. 11. A plot of (the moduli of) the normalized radial wave functions $\tilde{\varphi}\left(k_{r}, r\right)$ and $\varphi(-i \kappa, r)$ that include and exclude annihilation effects respectively. The inset details their behavior for small $r$. Notice that $\left|\tilde{\varphi}\left(k_{r}, r\right)\right|$ "heals" to $\varphi(-i \kappa, r)$ within $0.1 \mathrm{fm}$ away from the origin.

lytical solution given by Eq. (A10) for the phase equivalent Bargmann potential. Except in the vicinity of the origin, where the numerical solution is underestimated by a factor $\sim 1.2$, the two wave functions are essentially identical. Also shown is the wave function at $10 \mathrm{MeV}$ binding in a Gaussian potential that was used in [45] to estimate $\psi(0)$.

Finally in Fig. 10 we illustrate the variation of the spatial wave function $\psi(0)$ of Eq. (A10) as a function of its binding energy. This plot was obtained by using the calculated variation of $a_{0}, r_{0}$ and $\varepsilon$ with cutoff as shown in Fig. 2 for the one-boson exchange potential to obtain the parameters $a$ $=-\kappa$ and $b$ at each binding energy.

The inclusion of the annihilation potential changes the solution for the radial eigenfunction from $\varphi(-i \kappa, r)$ to the complex solution

$$
\tilde{\varphi}\left(k_{r}, r\right)=f\left(k_{r}, r\right) / f\left(k_{r}, 0\right)
$$

where $k_{r}=(0.1254-0.1972 i) M_{K}$ is the root of the Jost function in the presence of annihilation as given by Eq. (50). One readily verifies that $\tilde{\varphi}\left(k_{r}, 0\right)=1$ and $\tilde{\varphi}^{\prime}\left(k_{r}, 0\right)=\zeta=-i \xi$ as required. A comparative plot of the normalized versions of the moduli of $\tilde{\varphi}\left(k_{r}, r\right)$ and $\varphi(-i \kappa, r)$ is given in Fig. 11. Since the derivative of $\tilde{\varphi}\left(k_{r}, r\right)$ at the origin is purely imaginary, its modulus has a zero derivative there. This is clearly visible in the inset in Fig. 11, that also shows how $\left|\tilde{\varphi}\left(k_{r}, r\right)\right|$ "heals" within $0.1 \mathrm{fm}$ away from the origin from the effects of the annihilation potential to rejoin the real solution $\varphi$ $(-i \kappa, r)$. Either eigenfunction leads to a rms molecular radius of $\sqrt{\left\langle r^{2}\right\rangle} \approx 1.62 \mathrm{fm}$ (i.e. about two proton charge radii).

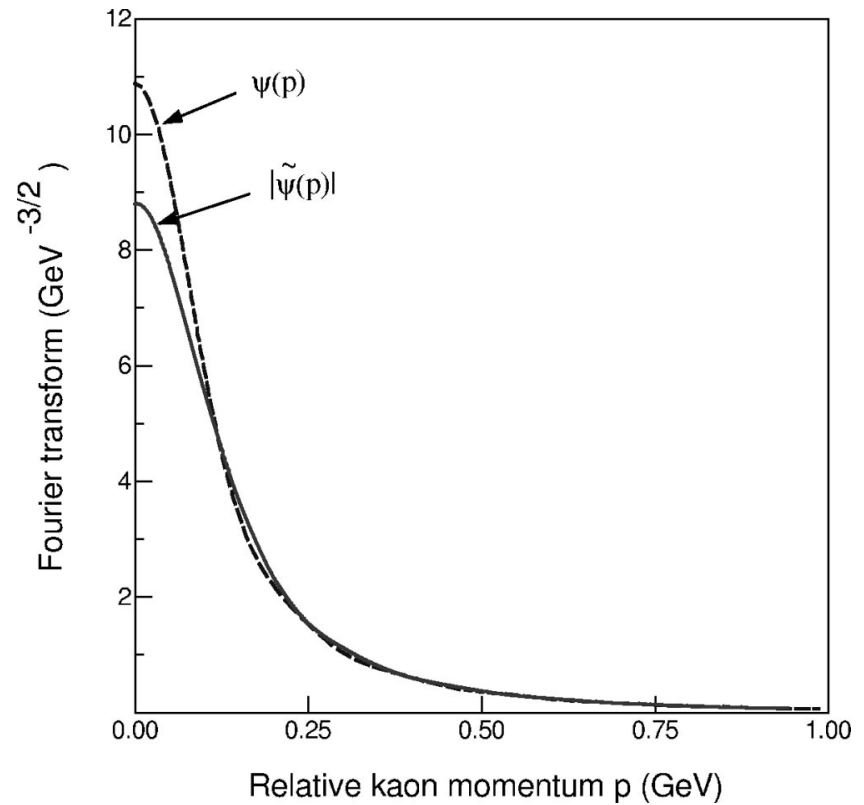

FIG. 12. Normalized Fourier transforms of the spatial wave functions $\widetilde{\psi}(r)$ with and $\psi(r)$ without annihilation.

The momentum content of the normalized spatial wave functions $\quad \psi(r)=(\sqrt{4 \pi} r)^{-1} \varphi(-i \kappa, r)$ and $\widetilde{\psi}(r)$ $=(\sqrt{4 \pi} r)^{-1} \tilde{\varphi}\left(k_{r}, r\right)$ is given by their Fourier transforms $\psi(p)$ and $\widetilde{\psi}(p)$. These are shown in Fig. 12 for relative meson momenta up to $1 \mathrm{GeV}$. As expected from Fig. 11, the effect of the annihilation potential is to redistribute the momentum to higher values, while at the same time leaving the characteristic value of $\left\langle p^{2}\right\rangle$ at $\sim 0.08 \mathrm{GeV}^{2}$ for the relative meson momentum squared in the molecule.

\section{Matching of isospin amplitudes}

The wave functions of kaonium in the inner, or isospin conserving region are given by Eq. (A5). These solutions either vanish at the origin, or possess a given logarithmic derivative there, depending on the form of $f(k)$ that is chosen. Matching either form onto the linear combinations $\varphi_{p}$ $\pm \varphi_{n}$ of the outside solutions at $r=d$, one finds the consistency condition

$$
\left(\begin{array}{cc}
\zeta_{0}-\zeta_{p} & \zeta_{0}-\zeta_{n} \\
\zeta_{1}-\zeta_{p} & -\zeta_{1}+\zeta_{n}
\end{array}\right)\left(\begin{array}{l}
\varphi_{p} \\
\varphi_{n}
\end{array}\right)=0
$$

where $\zeta_{n}=\varphi_{n}^{\prime} / \varphi_{n}$. The other logarithmic derivatives have been defined in the main text. At momenta $k \sim \mu \alpha$ of relevance for kaonium, $\zeta_{n}=i k$ is much smaller than any of these so we simply drop it and find

$$
\frac{1}{\zeta_{p}} \approx \frac{1}{2}\left(\frac{1}{\zeta_{0}}+\frac{1}{\zeta_{1}}\right) ; \quad \varphi_{n} / \varphi_{p} \approx-1+\zeta_{p} / \zeta_{0}=1-\zeta_{p} / \zeta_{1}
$$

The eigenvalue condition $\zeta_{p}=\zeta_{c}$ determines the values of $k$ $=-i \kappa$, which are pure imaginary in the absence of absorption, for the bound states of kaonium, and at the same time removes the outgoing wave piece of $\varphi_{p}$ from Eq. (69). Then 
the matching conditions show that the kaonium eigenfunctions that join smoothly onto the pure decaying state $f_{c}^{(-)}(-i \kappa r) \sim W_{1 / \lambda, 1 / 2}(2 \kappa r)$ outside the strong potential are given by a linear combination of isospin zero and one internal functions as

$$
\begin{gathered}
\varphi_{1 / \lambda}(r)=\frac{1}{2} \zeta_{c} f_{c}\left[\frac{1}{\zeta_{0}} \frac{\varphi_{0}(-i \kappa, r)}{\varphi_{0}(-i \kappa, d)}+\frac{1}{\zeta_{1}} \frac{\varphi_{1}(-i \kappa, r)}{\varphi_{1}(-i \kappa, d)}\right] \\
r \leqslant d=f_{c}(-i \kappa, r), \quad r \geqslant d
\end{gathered}
$$

where $\zeta_{c}=\zeta_{c}(-i \kappa)$ and $f_{c}=f_{c}^{(-)}(-i \kappa, d)$. We have labeled these eigenfunctions with the "quantum number" $\lambda^{-1}$ $=\mu \alpha / \kappa$ that reverts to an integer for a pure Coulomb field. One verifies immediately that the matching of value at $r$ $=d$ is guaranteed by the eigenvalue condition (74) or Eq. (A12), while the derivatives match identically from the definition of the logarithmic derivatives.

These eigenfunctions develop a common additional node at the $K^{+} K^{-}$scattering length, independent of the energy of the kaonium level. Consider the case where the scattering length is real. Then this happens because $\kappa \sim \mu \alpha \ll M_{K}$ is very small so that the behavior of the $\varphi_{I}(-i \kappa, r)$ differ but little from their zero energy behavior. From Eq. (A7) this is

$$
\begin{aligned}
\varphi(0, r)= & \frac{b-a}{b^{2}}\left\{\frac{(b+a) \sinh (b r)}{b \cosh (b r)+a \sinh (b r)}\right. \\
& \left.+r \frac{b a}{b-a} \frac{b \sinh (b r)+a \cosh (b r)}{b \cosh (b r)+a \sinh (b r)}\right\} \\
\sim & r, \quad b r \ll 1 \\
\sim & \frac{a}{b}\left(r-a_{I}\right), \quad b r \gg 1
\end{aligned}
$$

where $a$ and $b$ refer to channel $I$. Inserting this information into Eq. (A13), one finds that

$$
\varphi_{1 / \lambda} \sim-\left(\zeta_{c} a_{p}\right) f_{c}\left(1-r / a_{p}\right)
$$

outside the range of the strong interaction.

We return to the neglect of the $K^{0}-K^{ \pm}$mass difference $\Delta$ in calculating the logarithmic derivative $\zeta_{n}$ for the $K^{0} \bar{K}^{0}$ channel. Including $\zeta_{n}=-\sqrt{2 M_{K} \Delta}$ leads to a revised version of the eigenvalue equation (74) that now reads

$$
1-\frac{1}{2}\left(\zeta_{c}+\zeta_{n}\right)\left(\frac{1}{\zeta_{0}}+\frac{1}{\zeta_{1}}\right)+\frac{\zeta_{c} \zeta_{n}}{\zeta_{0} \zeta_{1}}=0
$$

The lowest eigenvalue of this equation is $\lambda=0.9860$ $+0.0061 i$ instead of $0.9863+0.0082 i$, an inessential change. The reason is the dominating influence of the isoscalar channel for which $\zeta_{0} d \approx 18.0-10.5 i$, that overshadows the remaining logarithmic derivatives, which are all $O(1)$, and suppresses the last term in Eq. (A16).

\section{Coulomb distortion}

It is also useful to document the modification due to the Coulomb interaction on the low-momentum behavior of the absorption cross section as given by the third of Eqs. (65). Using Eqs. (71), (72) and (75), one finds that

$\sigma_{a} \approx \frac{4 \pi}{k^{2}} \operatorname{Im}\left(a_{p}^{\text {eff }}\right) \operatorname{Im}\left(\zeta_{c}\right) \approx-\frac{4 \pi}{k} \frac{\operatorname{Im}\left(a_{p}^{\text {eff }}\right)}{\left|f_{c}^{(-)}(k, d)\right|^{2}}$

since $\operatorname{Im}\left(\zeta_{c}\right)=-k\left|f_{c}^{(-)}(k, d)\right|^{-2}$ is the imaginary part of the logarithmic derivative $\zeta_{c}$ of the incoming Coulomb wave. The latter relation is easily found by writing $f_{c}^{(-)}(k, r)$ $=\exp (i \sigma)[G(k, r)-i F(k, r)]$ in terms of the regular and irregular Coulomb solutions $F(k, r) \sim \sin (k r-\eta \ln 2 k r+\sigma)$, $G(k, r) \sim \cos (k r-\eta \ln 2 k r+\sigma)$, so that

$$
\zeta_{c}=\left(\frac{G G^{\prime}+F F^{\prime}-i k}{G^{2}+F^{2}}\right)_{r=d}
$$

after using the Wronskian relation $\left(G F^{\prime}-G^{\prime} F\right)=k$ that holds for all $r$. For $k d \sim \mu \alpha d \ll 1$ one finds from Eq. (70) that

$$
\begin{gathered}
\left|f_{c}^{(-)}(k, d)\right|^{2}=\left(\frac{e^{2 \pi \eta}-1}{2 \pi \eta}\right)\left[[1-2 \mu \alpha d(\ln 2 \mu \alpha d+2 \gamma-1)]^{2}\right. \\
\left.+\left(\frac{2 \pi \mu \alpha d}{\left(e^{2 \pi \eta}-1\right)}\right)^{2}\right] \sim\left(\frac{e^{2 \pi \eta}-1}{2 \pi \eta}\right), \\
\eta=-\mu \alpha / k
\end{gathered}
$$

in agreement with Ref. [55].

\section{Detailed balance}

If one ignores the real one-boson exchange potential $V_{I}$ in Eq. (33), then the Jost function for $K \bar{K}$ scattering due to the annihilation part alone is simply $f(k)=\zeta+i k$. This gives rise to an $s$-wave $I=0$ scattering length of

$$
4 \pi a_{0}(K \bar{K})=-M_{K} c_{0}^{2}
$$

after using the definition (34) for $\zeta$. However, since $\pi \pi$ annihilation into $K \bar{K}$ is described by the same diagram in Fig. 3 with the pion and kaon lines interchanged, the $I$ $=0, \pi \pi$ scattering length due to this process alone is also given to the same approximation by the expression as above, but with $M_{\pi}$ and $M_{K}$ interchanged in the product $M_{K} c_{0}^{2}$, i.e.

$$
\begin{aligned}
4 \pi a_{0}(\pi \pi) & =-\left[M_{K} c_{0}^{2}\right]_{M_{\pi} \leftrightarrow M_{K}}=-\frac{k}{p} M_{K} c_{0}^{2} \\
& =4 \pi a_{0}(K \bar{K})\left(\frac{k}{p}\right) .
\end{aligned}
$$

We have used Eq. (31) to perform the mass interchange. Here $p$ and $k$ are the relative momenta of a pion or kaon pair with the same total CM energy $P_{0}$. There is no contribution to the $a_{2}(\pi \pi)$ scattering length from Fig. 3 in the $I=2$ 
channel, since the intermediate kaon loop can at most carry $I=1$. Consequently detailed balance holds between the $I$ $=0$ absorption cross section $\sigma_{a}^{(0)}(\pi \pi \rightarrow K \bar{K})$ and its inverse $\sigma_{a}^{(0)}(K \bar{K} \rightarrow \pi \pi)$ when viewed in the isospin basis under the above approximations.

However the physical $\pi^{+} \pi^{-}$channel is described by the scattering length $a\left(\pi^{+} \pi^{-}\right)=\frac{2}{3} a_{0}(\pi \pi)+\frac{1}{3} a_{2}(\pi \pi)$. But $\operatorname{Im}\left[a_{2}(\pi \pi)\right]=0$, so

$$
\begin{aligned}
\sigma_{a}\left(\pi^{+} \pi^{-} \rightarrow K \bar{K}\right) & =-\frac{4 \pi}{p} \operatorname{Im}\left(\frac{2}{3} a_{0}(\pi \pi)\right) \\
& =\frac{2}{3} \frac{k^{2}}{p^{2}} \sigma_{a}^{(0)}(K \bar{K} \rightarrow \pi \pi)
\end{aligned}
$$

provided that $P_{0} \geqslant 2 M_{K}$.

\section{Dimensional regularization of $J\left(P_{0}\right)$}

Replace the three-dimensional integral in Eq. (29) by its analog in $d$ dimensions [16],

$$
\begin{aligned}
J\left(P_{0}\right) & =\int \frac{d^{3} \mathbf{I}}{(2 \pi)^{3}}\left(\frac{1}{\mathbf{l}^{2} / M+2 M-P_{0}}\right) \\
& \rightarrow \frac{1}{(4 \pi)^{d / 2}} \frac{1}{\Gamma(d / 2)} \int_{0}^{\infty} d L^{2} \frac{\left(L^{2}\right)^{d / 2-1}}{L^{2} / M+2 M-P_{0}} \\
& =\left[M\left(2 M-P_{0}\right)\right]^{d / 2-1} \frac{M}{(4 \pi)^{d / 2}} \Gamma[1-d / 2]
\end{aligned}
$$

or, as $d \rightarrow 3$,

$$
\begin{aligned}
J\left(P_{0}\right)= & -\frac{M}{4 \pi}\left[M\left(2 M-P_{0}\right]^{1 / 2}\right. \\
= & \frac{M}{4 \pi}\left[M\left|\left(P_{0}-2 M\right)\right|\right]^{1 / 2} e^{i(\phi+\pi) / 2} \\
& 0<\phi<2 \pi J P
\end{aligned}
$$

on the first sheet of the complex $P_{0}$ plane. Note that $J\left(P_{0}\right)$ is pure imaginary and positive on the upper lip of the cut $\phi$ $=0$ extending from $2 M$ to $\infty$. The analytic continuation to the second sheet through the cut starting at the branch point $P_{0}=2 M$ is obtained by taking $-2 \pi<\phi<0$.
[1] C. Amsler, Rev. Mod. Phys. 70, 1293 (1998).

[2] S. Godfrey and J. Napolitano, Rev. Mod. Phys. 71, 1411 (1999).

[3] L. Montanet, Nucl. Phys. B (Proc. Suppl.) 86, 381 (2000).

[4] V.V. Anisovich et al., Phys. Lett. B 480, 19 (2000).

[5] N.N. Achasov and V.N. Ivanchenko, Nucl. Phys. B315, 465 (1989).

[6] J. Weinstein and N. Isgur, Phys. Rev. Lett. 48, 659 (1982); Phys. Rev. D 27, 588 (1983); 41, 2236 (1990).

[7] M.N. Achasov et al., Phys. Lett. B 485, 349 (2000).

[8] A. Aloisio et al., Phys. Lett. B 537, 21 (2002).

[9] J.A. Oller, Nucl. Phys. A714, 161 (2003).

[10] N.N. Achasov, hep-ph/0309118.

[11] GAMS Collaboration, D. Alde et al., Z. Phys. C 66, 375 (1995).

[12] E852 Collaboration, J. Gunter et al., Phys. Rev. D 64, 072003 (2001).

[13] N.N. Achasov et al., Phys. Rev. D 58, 054011 (1998).

[14] F.P. Sassen et al., Phys. Rev. D 68, 036003 (2003).

[15] E. Lipartia, V.E. Lyubovitskij, and A. Rusetsky, Phys. Lett. B 533, 285 (2002).

[16] J. Gasser, V.E. Lyubovitskij, A. Rusetsky, and A. Gall, Phys. Rev. D 64, 016008 (2001).

[17] For a recent review, see "Proceedings of the International Workshop on Hadronic Atoms," CERN, Geneva, 2002, edited by L. Afanasyev, A. Lanaro, and J. Schacher, hep-ph/0301266.

[18] A. Czarnecki, K. Melnikov, and A. Yelkhovsky, Phys. Rev. A 59, 4316 (1999).

[19] J.J. de Swart, Rev. Mod. Phys. 35, 916 (1963).

[20] D. Lohse, J.W. Dorso, K. Holinde, and J. Speth, Nucl. Phys. A516, 513 (1990).
[21] G. Janßen, B.D. Pearce, K. Holinde, and J. Speth, Phys. Rev. D 52, 2690 (1995).

[22] K. Kawarabayashi and M. Susuki, Phys. Rev. Lett. 16, 255 (1966); X. Riazuddin and X. Fayyazuddin, Phys. Rev. 147, 1071 (1966).

[23] F. P. Sassen, Diplomarbeit, Rheinischen Freidrich-WilhelmsUniversität, Bonn, 2001.

[24] V. Bargmann, Rev. Mod. Phys. 21, 488 (1949).

[25] R.G. Newton, Phys. Rev. 105, 763 (1956).

[26] Fermilab E791 Collaboration, E.M. Aitala et al., Phys. Rev. Lett. 86, 765 (2001).

[27] S.D. Protopopescu et al., Phys. Rev. D 7, 1279 (1973).

[28] A.D. Martin et al., Nucl. Phys. B121, 514 (1977).

[29] B. Hyams et al., Nucl. Phys. B64, 134 (1973).

[30] O. Krehl, R. Rapp, and J. Speth, Phys. Lett. B 390, 23 (1997).

[31] A.E. Kudryavtsev and V.S. Popov, JETP Lett. 29, 280 (1979); V.S. Popov, A.E. Kudryavtsev, and V.D. Mur, Sov. Phys. JETP 50, 865 (1979).

[32] See, for example, L. D. Landau and E. M. Lifshitz, Relativistic quantum theory, Course of Theoretical Physics Vol. 4 (Pergamon, Oxford, 1974).

[33] The standard graphical representation of the BS equation is given in Ref. [32], to which we refer the reader for further details.

[34] R.G. Newton, J. Math. Phys. 1, 319 (1960).

[35] This is perhaps the simplest choice out of an entire family of phase equivalent potentials (the case $c=2$ in the notation of [34]).

[36] This can also be interpreted as $\varphi(k, r)$ behaving discontinuously at the origin, $\varphi(k, 0)=0$, but $\lim \varphi(k, r) \neq 0$ as $r \rightarrow 0$ 
under the influence of the delta function potential.

[37] S. Deser, M.L. Goldberger, K. Baumann, and W. Thirring, Phys. Rev. 96, 774 (1954).

[38] J.L. Peterson, Phys. Rep. 2C, 151 (1971).

[39] For the values of $a$ and $b$ and the logarithmic derivatives given in Table I, it turns out that there is only one such root in the isoscalar channel, and none in the isovector channel.

[40] D. Morgan and M.R. Pennington, Phys. Lett. B 258, 444 (1991); Phys. Rev. D 48, 1185 (1993); M. Boglione and M.R. Pennington, ibid. 65, 114010 (2002).

[41] Note: these authors use the opposite convention for the definition of the Jost function to that employed in [34]. Thus their sheet II for complex $k$ corresponds to $\operatorname{Im} k \leqslant 0$ in our notation.

[42] Particle Data Group, K. Hikasa et al., Phys. Rev. D 45, S1 (1992).

[43] R.H. Lemmer and R. Tegen, Phys. Rev. C 66, 065202 (2002).

[44] C. Itzykson and J. Zuber, Quantum Field Theory (McGrawHill, New York, 1980).

[45] T. Barnes, Phys. Lett. 165B, 434 (1985).

[46] Particle Data Group, K. Hagiwara et al., Phys. Rev. D 66, 010001 (2002).

[47] N.N. Achasov, S.A. Devyanin, and G.N. Shestakov, Phys. Lett. 108B, 134 (1982); N.N. Achasov, Phys. Usp. 41, 1149 (1998).

[48] D. Robson, Phys. Rev. 137, 535 (1965).
[49] R. H. Lemmer, Cargese Lectures in Physics, edited by M. Jean (Gordon and Breach, New York, 1969), Vol. 3, p. 483.

[50] See, for example, R. G. Newton, Scattering Theory of Waves and Particles (McGraw-Hill, New York, 1966).

[51] See, for example, I. S. Gradshteyn and I. M. Ryzhik, Table of Integrals, Series, and Products (Academic, New York, 1980).

[52] This channel is actually closed and decays exponentially like $\sim \exp \left(-\sqrt{2 M_{K^{0}} \Delta} r\right)$ when isospin breaking due to the $K^{0}-K^{ \pm}$ mass difference $\Delta \sim 4 \mathrm{MeV}$ is not ignored. This is an inessential approximation in the presence of the dominating isoscalar channel. Keeping $\Delta$ changes the eigenvalues of Eq. (77) by at most $\sim 10 \%$, see below Eq. (A16).

[53] S. Wycech and A.M. Green, Nucl. Phys. A562, 446 (1993); S.V. Bashinsky and B.O. Kerbikov, Phys. At. Nucl. 59, 1979 (1996).

[54] The predicted lifetime of kaonium is three orders of magnitude shorter than that for the pionium $\rightarrow 2 \pi^{0}$ decay [16]. Apart from specific details of the annihilation interaction for pionium, its decay rate is slowed down by the relatively small mass difference $M_{\pi^{+}}-M_{\pi^{0}} \sim 5 \mathrm{MeV}$ that drives the decay as opposed to $M_{K}-M_{\pi} \sim 356 \mathrm{MeV}$ for kaonium. Also, the $\pi \pi$ system has no bound state analogous to $K \bar{K}$.

[55] L.D. Landau and E. M. Lifshitz, Quantum Mechanics, Vol. 3 (Pergamon, London, 1958). 